\title{
Clave para la Identificación de las Especies de Murciélagos de Honduras
}

\author{
José Manuel Mora ${ }^{1}$
}

\begin{abstract}
Resumen. La diversidad de murciélagos de Honduras, aunque es muy rica y variada, recibió relativamente poca atención hasta recientemente. Nuevas actividades de desarrollo económico y nuevas necesidades de conocimiento, junto con una nueva generación de biólogos de campo, están impulsando la investigación de los murciélagos del país. Debido a esto, se elaboraró una clave de campo para la identificación de los murciélagos de Honduras. El documento contiene: una clave para la separación e identificación de las ocho familias de murciélagos de Honduras, una clave para la separación e identificación de las ocho subfamilias de Phyllostomidae, siete claves individuales para separar las especies dentro de las familias y ocho claves individuales para separar las especies dentro de las subfamilias de Phyllostomidae. En total se han incluido 114 especies de murciélagos, 110 registradas para Honduras más cuatro adicionales esperadas (sin registro oficial) para el país. La clave se ha basado en el conocimiento y documentos generales generados para la identificación de los murciélagos de otros países de la región. A la vez, se ha incluido la literatura reciente en este tema para incorporar las modificaciones recientes en la taxonomía de las especies y clasificación de taxa superiores.
\end{abstract}

Palabras clave: Emballonuridae, Molossidae, Mormoopidae, Natalidae, Noctilionidae, Phyllostomidae, Thyropteridae, Vespertilionidae.

\section{Identification Key to the Species of Bats of Honduras}

\begin{abstract}
The diversity of Honduras bats, although very rich and varied, received relatively little attention until recently. The economic development and new knowledge needs, along with a new generation of field biologists, are guiding the research of bats in the country. Due to these facts, a field key for Honduras bats was elaborated. The document contains: a key to separate and identify the eight families of bats present in Honduras, a key to separate and identify the eight subfamilies of Phyllostomidae, seven individual keys to separate the species within each family and eight individual keys to separate the species within each Phyllostomidae subfamily. The key includes 114 species of bats, 110 registered for Honduras plus four additional expected (without official registration) for the country. The key is based on general and specific documents generated for the identification of bats from other countries in the region. At the same time, current literature on this topic has been included to incorporate recent changes in species taxonomy and higher taxa classification.
\end{abstract}

Key words: Emballonuridae, Molossidae, Mormoopidae, Natalidae, Noctilionidae, Phyllostomidae, Thyropteridae, Vespertilionidae.

\section{Introducción}

La manera de identificar en el campo a las especies de murciélagos de Centro América, al menos para muchos biólogos no expertos en quirópteros, cambió con la llegada del libro de Fiona Reid "Mammals of Central America and southeast Mexico" $(1997,2009)$. El texto y las ilustraciones en este libro son una ayuda invaluable en esta labor. No obstante, no todos los trabajadores de campo tienen acceso al libro o son capaces de leer el inglés. El libro tampoco constituye o es una clave de identificación por lo que la identificación de las especies a veces se complica. Esta es la situación que ocurre comúnmente en Honduras con los murciélagos. Adicionalmente, en el país no se ha contado con una clave de campo que ayude en esta labor. En vez de esto, los biólogos locales han dependido en el campo del uso de claves o al menos las listas de especies de otros países de la región, particularmente Costa Rica, Guatemala y

\footnotetext{
${ }^{1}$ Instituto Internacional en Conservación y Manejo de Vida Silvestre (ICOMVIS), Universidad Nacional (UNA), Heredia, Costa Rica. Correo electrónico josemora07@gmail.com
} 
México. El conocimiento de los murciélagos de esos países es mayor que en Honduras. Particularmente en Centro América, este conocimiento ha sido generado por el trabajo de investigadores extranjeros principalmente, hasta recientemente en que una nueva y mayor generación de biólogos se ha inclinado a trabajar con murciélagos en la región, especialmente en conservación (Rodríguez-Herrera y Sánchez 2015).

En Costa Rica ha existido una larga tradición de investigación biológica, un departamento de biología desde 1957 (García 2009) y la Organización de Estudios Tropicales (OET) desde 1963. La OET es un consorcio de universidades que ha dictado cursos relacionados a la biología tropical con el apoyo de grandes expertos mundiales en diversos campos. Los murciélagos no han sido la excepción y, ya desde 1971, el país contó con una clave para la identificación de murciélagos de Costa Rica elaborada por Andrew Starrett incluida en una recopilación de información hecha por la OET y conocida como "The Book" (OTS 1971). Esa clave fue usada, probada, mejorada y ampliada por varios investigadores hasta que Timm y LaVal (1998) publicaron "A field key to the bats of Costa Rica", la cual fue traducida y publicada en español por Timm et al. (1999). En Guatemala particularmente el trabajo de Tim McCarthy del Carnegie Museum contribuyó enormemente al conocimiento de los murciélagos del país. Precisamente este último autor, junto con otros coautores, incluido el biólogo hondureño Gustavo Cruz, publicaron un trabajo con los registros, los recolectores y las listas de las especies de murciélagos del norte de Centroamérica, incluida Honduras (McCarthy et al. 1993).

Durante casi dos décadas, casi nada adicional sucedió sobre los medios para la identificación de los murciélagos de Honduras y muy poco para su conocimiento particular, excepto por supuesto el libro de Reid $(1997,2009)$ ya referido. Marineros y Martínez (1998) recopilaron información general sobre los mamíferos de Honduras y señalaron la existencia de 98 especies de murciélagos en el país basados principalmente en McCarthy et al. (1993). Sin embargo, Marineros y Martínez (1998) proveyeron la información de solo 85 especies dentro del texto. La lista de McCarthy et al. (1993) incluyó 99 especies de murciélagos para Honduras. A esta lista Reid (2009) adicionó Hylonycteris underwoodi y Diaemus youngii. Al tomar en cuenta los cambios taxonómicos y nuevos registros de distribución, el número de especies en
Honduras era de 101 en 2009. Del 2009 al 2016 se adicionaron dos especies debido a evaluaciones de taxonomía. Se adicionaron Rhogeessa menchuae y $R$. bickhami y se eliminó a $R$. tumida (Baird et al. 2012). Se adicionaron Uroderma davisi y $U$. convexum y se eliminó a U. bilobatum (Mantilla-Meluk 2014). Hubo tres registros basado en muestreos con redes de niebla, Lasiurus egregius (Mora 2012), Balantiopteryx io (Divoll y Buck 2013) y Vampyriscus nymphaea (Mora et al. 2014). Además hubo tres nuevos reportes basado en individuos encontrados muertos en parques eólicos Lasiurus cinereus (Mora y López 2012), Nyctinomops aurispinosus (Espinal et al. 2016) y Nyctinomops macrotis (Mora et al. 2016). Una adición más fue Eptesicus brasiliensis, detectado por medios acústicos y también capturado en redes de niebla (Espinal y Mora 2016). Estas 110 especies y algunas más que casi de seguro existen en el país, son un rico elemento para la biodiversidad de Honduras y su conocimiento debe ser fomentado y divulgado. Con el advenimiento de un grupo cada vez mayor de jóvenes biólogos nacionales se considera que una clave será una enorme ayuda que apoye su trabajo de identificación de las especies en el campo.

Vistos todos los factores anteriores, se ha preparado una clave para la identificación de los murciélagos de Honduras la cual debe ser validada por múltiples trabajadores de campo. Esta clave está basada en la clave de los murciélagos de Costa Rica (Timm y LaVal 1998) y en la clave de los murciélagos de México (Medellín et al. 1997). Además, esta clave ha sido complementada con información de varias fuentes bibliográficas, especialmente por la guía de campo de los mamíferos de Centro América y sureste de México (Reid 2009). Esta última referencia, así como las dos claves mencionadas han sido las herramientas principales para la identificación de los murciélagos de Honduras para muchos investigadores de campo. Esta familiaridad, especialmente con la clave de los murciélagos de Costa Rica (Timm et al. 1999), ha sido otro factor influyente para basar esta clave de murciélagos en estas fuentes. Sin duda estos investigadores tienen una gran cuota de apoyo al conocimiento de los murciélagos de Honduras y su identificación. Es necesario actualizar la nomenclatura y clasificación de los murciélagos de Honduras debido a la gran cantidad de cambios que se han dado a nivel específico y en taxa mayores en los últimos años (e.g. Baird et al. 2015, Baker et al. 2016, Ziegler et al. 2016). Por ejemplo se ha propuesto que el género Lasiurus sea dividido en tres (Aeorestes, Dasypterus y 
Mora: Clave para la Identificación de las Especies de Murciélagos de Honduras

Lasiurus, Baird et al. 2015). Sin embargo, Ziegler et al. (2016) consideran que este cambio no es válido.

La clave incluye las 110 especies mencionadas y cuatro especies adicionales: Cormura brevirostris, Lampronycteris brachyotis, Trinycteris nicefori y Natalus lanatus. Las tres primeras especies fueron incluidas en la lista de murciélagos de Honduras dentro de la Estrategia Centroamericana para la Conservación de Murciélagos (Hernández 2015). El límite norte de la distribución conocida de Cormura brevirostris es la Moskitia nicaragüense, cerca de la Moskitia hondureña. Lampronycteris brachyotis y Trinycteris nicefori existen al norte y sur de Honduras por lo que se espera que también existan en el país. En adición de las especies esperadas incluidas por Hernández (2015), esta clave incluye a Natalus anatus, una especie descrita para México y recientemente reportada para Costa Rica (Rodríguez et al. 2011) y Nicaragua (Medina-Fitoria et al. 2014).

La clave incluye las principales características de las especies, por ejemplo sus generalidades (Figura 1), así como la ilustración de algunas características particulares. No obstante, la eficiencia de su uso depende de la preparación de los usuarios quienes deben tener un entrenamiento apropiado para la manipulación y el reconocimiento de los murciélagos.
Esto a la vez permitiría la mejora de la clave con su aplicación para encontrar aspectos que deben ser modificados para la identificación de las especies.

Algunos pares de especies tienen información adicional a la necesaria para la identificación de la especie o que de hecho es similar a su respectivo par. La idea es proveer a los usuarios de más datos pues no siempre poseen información adicional. Hay que erradicar la costumbre de algunos investigadores que al no ser capaces de identificar la especie, solo recolectan al animal para su posterior identificación. Muchos de estos animales son desechados sin aprovecharlos para colecciones de museo 0 de enseñanza. La idea de esta clave es contribuir al conocimiento de los murciélagos de Honduras y que a la vez este conocimiento sirva para la conservación de los murciélagos. Es deseable que los investigadores puedan identificar a las especies en el sitio y poder liberarlos tan pronto como hayan terminado con la respectiva toma de datos. Estoy seguro que esa ha sido y es la meta, como debe ser, de quienes han contribuido con las publicaciones, artículos, libros y claves para la identificación de los murciélagos, que se han mencionado antes y que se han incluido en la literatura citada de este trabajo. La meta de todos debe ser la conservación de los murciélagos y contribuir para ello al nivel posible de cada uno.

\section{Clave para las Familias de Murciélagos de Honduras}

1. Con discos de adhesión en los pulgares y en las patas (Figura 2) ......................... Thyropteridae

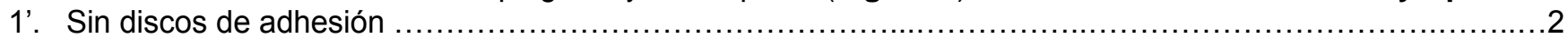

2. Con hoja nasal en forma de hoja o de lanza o de forma redondeada y rudimentaria o con surcos y pliegues (Figura 3) Phyllostomidae

2'. Sin hoja nasal 3

3. Cola contenida dentro del uropatagio, alcanza el borde o la punta se extiende fuera del borde del uropatagio (Figura 4 A)

3'. Cola libre, fuera del uropatagio (Figura 4 B) o sobresale por encima de la membrana (uropatagio) (Figura 4

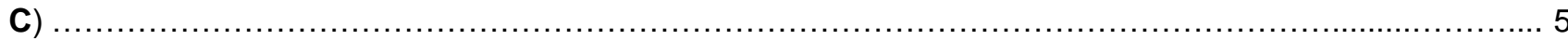

4. Orejas con forma de embudo (Figura 5); ojos muy pequeños; patas muy largas con la cola y el uropatagio también notoriamente alargados; dos falanges en el tercer dedo Natalidae

4'. Orejas sin forma de embudo; patas posteriores, cola, y uropatagio no notoriamente largos; tres falanges en el tercer dedo Vespertilionidae 


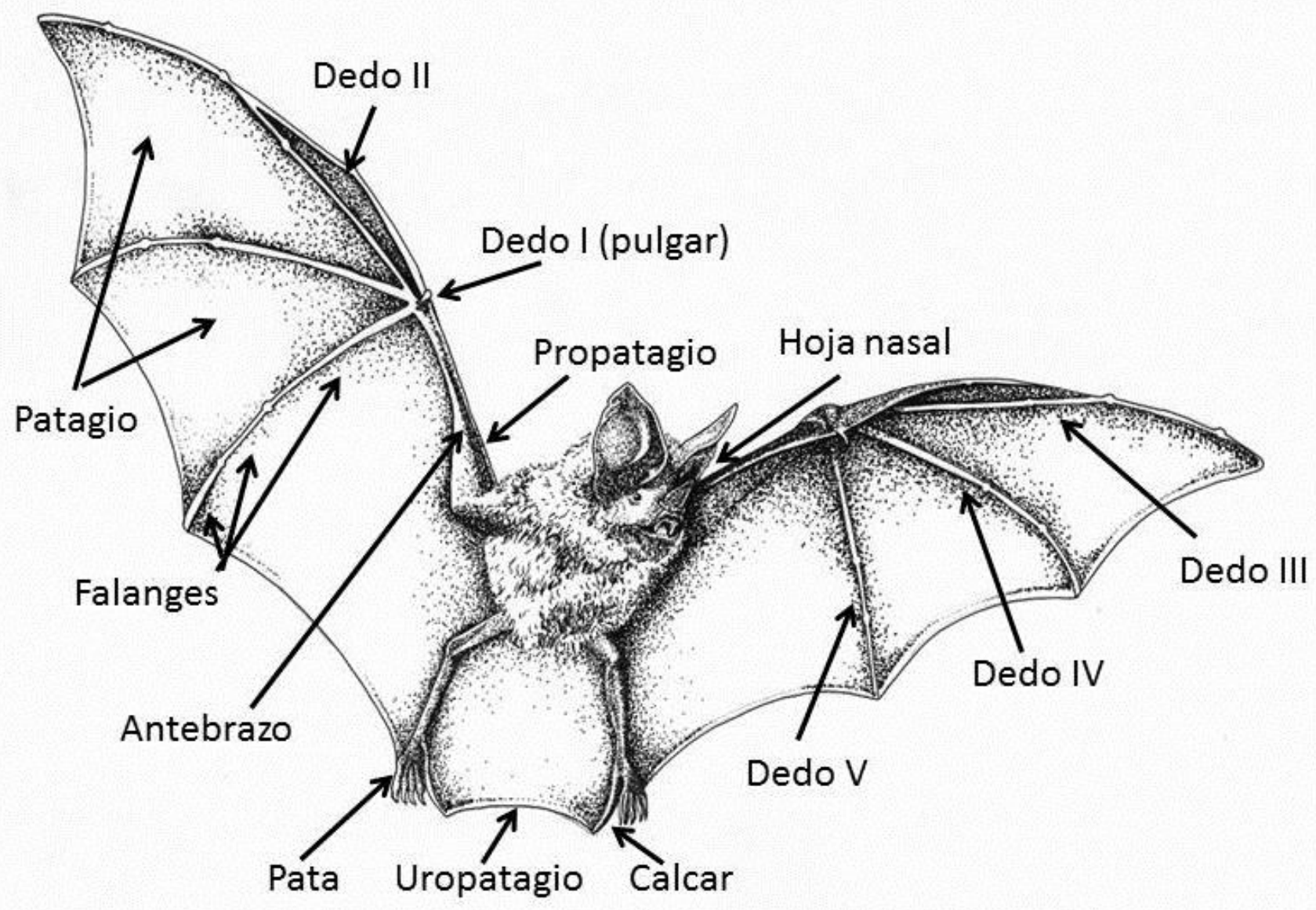

Figura 1. Características morfológicas externas generales de los murciélagos.
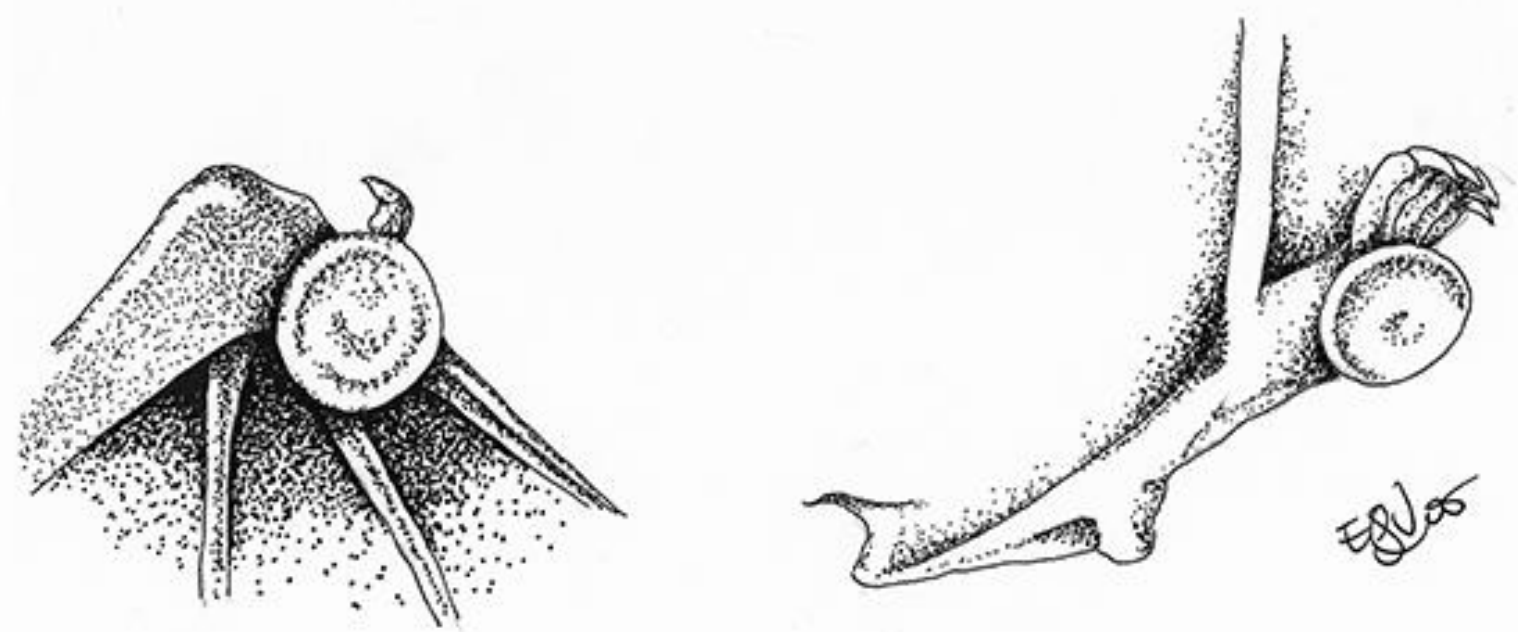

Figura 2. Disco de adhesión en la base del pulgar (izquierda) y en el pie (derecha) de Thyropteridae. 


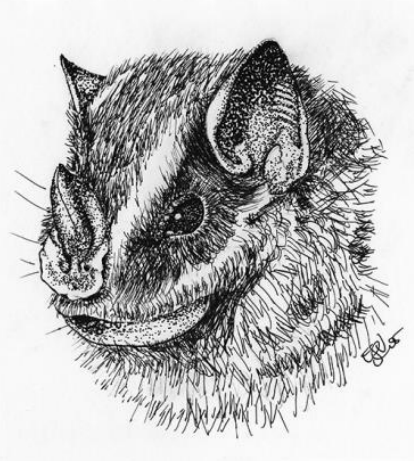

A

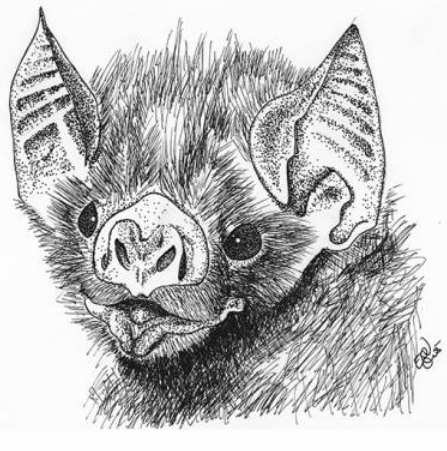

B

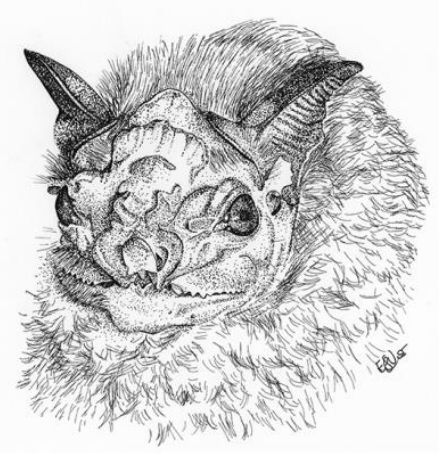

C

Figura 3. Hoja nasal en murciélagos filostómidos. A. Stenoderminae. B. Desmodus rotundus (Desmodontinae) con hoja nasal rudimentaria y redondeada. C. Centurio senex con surcos y pliegues.

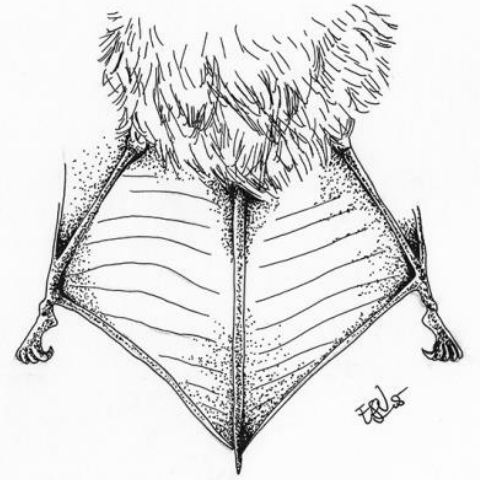

A

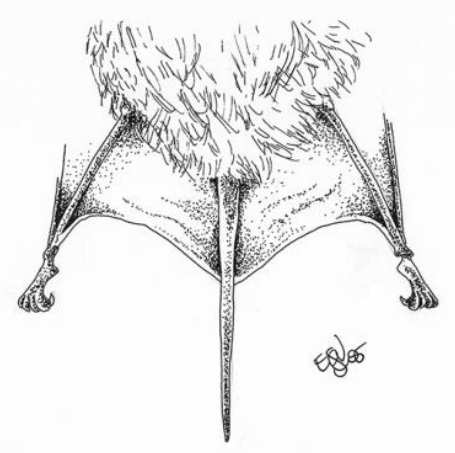

B

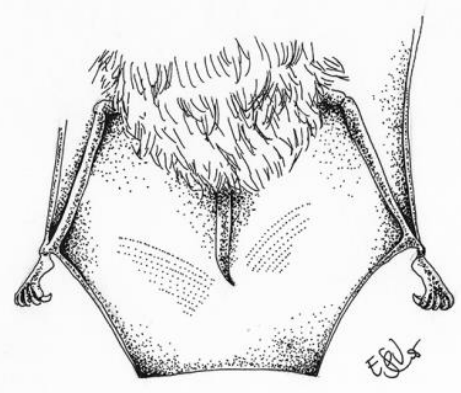

C

Figura 4. A. Cola contenida en el uropatagio. B. Cola fuera del uropatagio. C. Cola sobresale por encima del uropatagio.

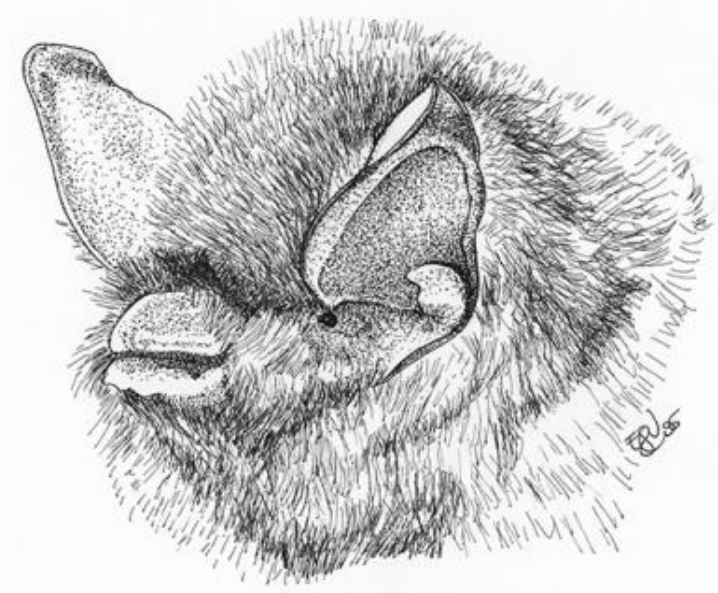

Figura 5. Natalus mexicanus (Natalidae). Note la forma característica de las orejas, así como otros rasgos del rostro. 
5. Cola gruesa y fuera del uropatagio (Figura 4 A)

Molossidae

5'. Cola sobresale por encima del uropatagio (Figura 4 C) 6

6. Rostro simple con labios y barbilla sin surcos, arrugas, o bordes (Figura 6); propatagio con bolsa en varias especies; el segundo dedo no tiene falanges

Emballonuridae

6'. Rostro con surcos, arrugas o bordes; sin bolsa en el propatagio; el segundo dedo tiene una o dos falanges....7

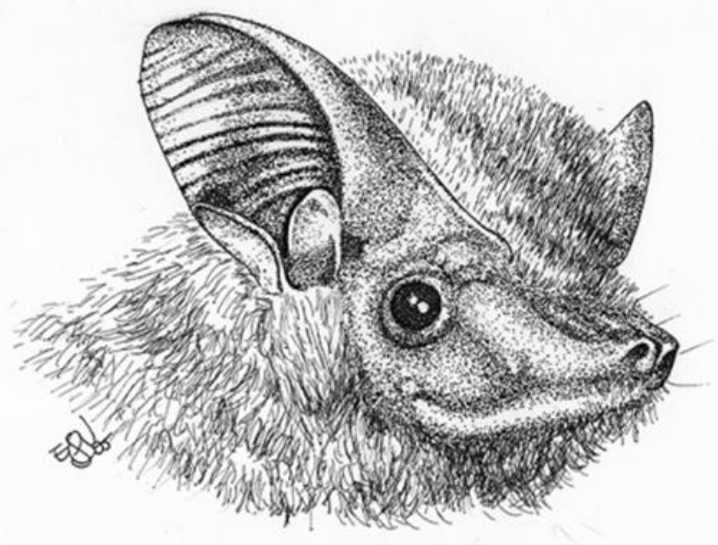

A

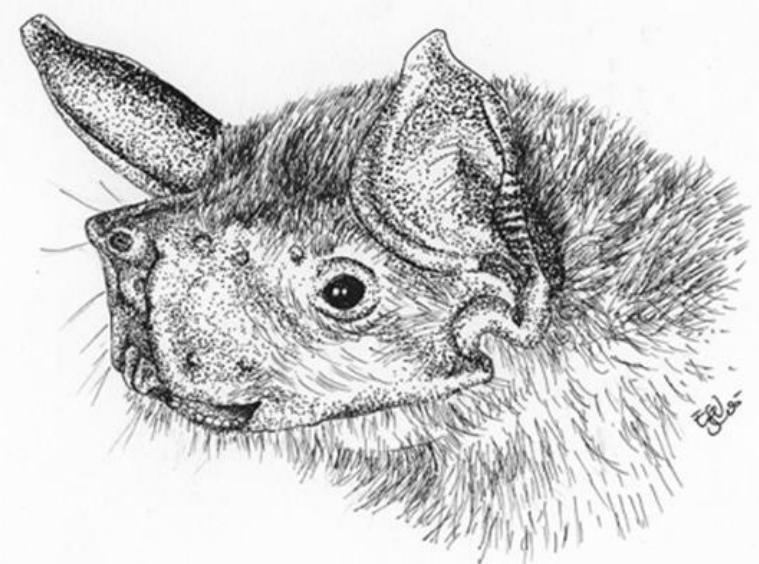

B

Figura 6. A. Rostro simple sin pliegues o arrugas en Emballonuridae y B. Rostro con hendiduras en el frente del rostro en Noctilionidae.

7. Labio superior dividido en el centro que le da un aspecto de labio leporino (Figura 6 B); labios sin adornos; dos incisos inferiores; el segundo dedo tiene dos falanges; pelaje muy corto Noctilionidae

7'. Labios con bordes, pliegues horizontales y con papilas pequeñas (Figura 7); cuatro incisos inferiores; segundo dedo tiene una falange; pelo largo y sedoso

Mormoopidae
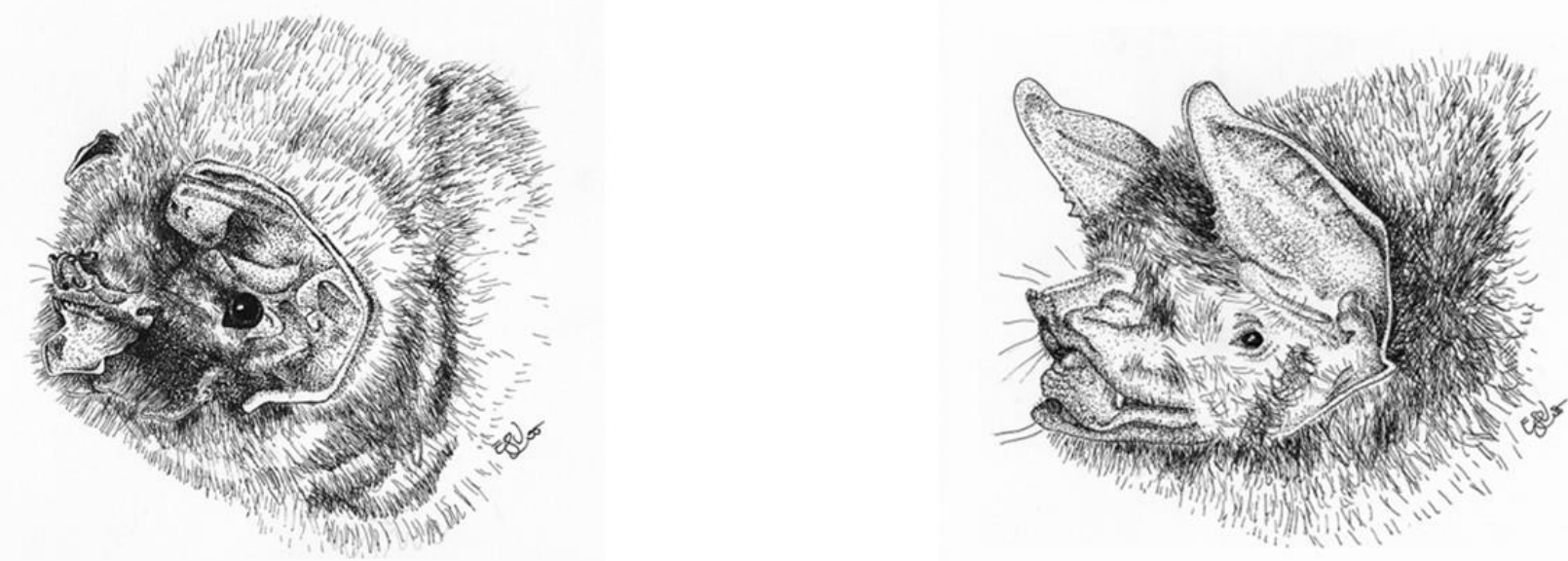

Figura 7. Arrugas y pliegues en Mormoopidae. Rostro de Mormoops (izquierda) y de Pteronotus (derecha). 


\section{Clave para las Especies de Emballonuridae (Murciélagos con Bolsas)}

1. Blanco; antebrazo $65-66 \mathrm{~mm}$; con una estructura en forma de $\vee$ queratinizada oscura en el uropatagio, notoria en los machos (Figura 8).

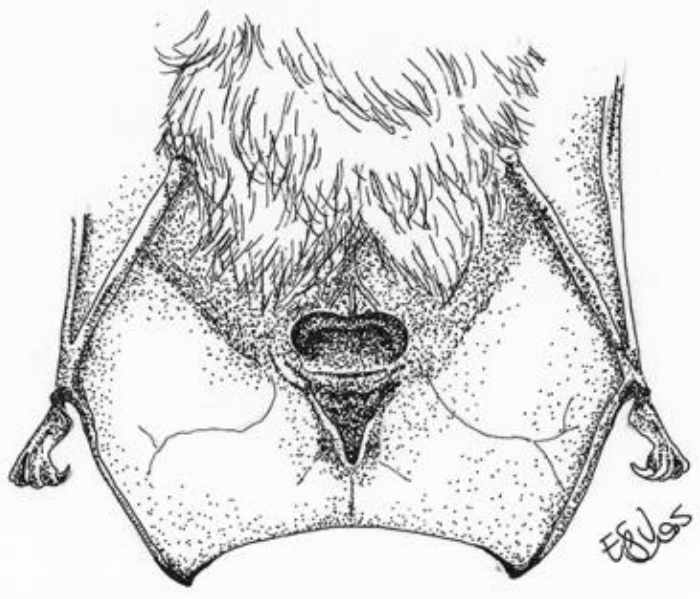

Figura 8. Estructura queratinizada en el dorso del uropatagio de Diclidurus albus.

2. Sin bolsa glandular en el propatagio

2'. Con una bolsa glandular en el propatagio, con la abertura en la superficie dorsal (Figura 9), poco evidente o apenas visible en las hembras.

3. Antebrazo $36-40 \mathrm{~mm}$; dos líneas pálidas en la espalda; pelaje barcino, amarillento o grisáceo; antebrazo con mechones notorios de pelo claro; labio superior y nariz alargados como una proboscis; orejas largas y puntiagudas

Rhynchonycteris naso

3' Antebrazo 43-49 mm; sin líneas blancas; pelaje amarillo pálido o café grisáceo, antebrazo sin mechones de pelo; labio superior y nariz no en forma de proboscis; orejas en forma de hoz

Centronycteris centralis

4. Dos líneas onduladas claras en la espalda; bolsa glandular cerca del antebrazo con apertura hacia el cuerpo (Figura 9A).

4'. Sin líneas; bolsa glandular grande o pequeña con abertura orientada hacia el cuerpo o hacia afuera (Figura 9)

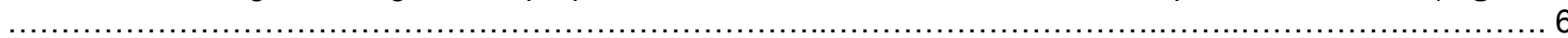

5. Antebrazo 44-48 mm; color negro o negro parduzco; líneas blancas o anteado Saccopteryx bilineata 5'. Antebrazo 37-43 mm; color café uniforme, líneas menos evidentes

6. Antebrazo $36-45 \mathrm{~mm}$; bolsa pequeña, en el centro del propatagio sin alcanzar su borde, con la abertura hacia el cuerpo (Figura 9B)

6'. Antebrazo 43-52 mm; bolsa pequeña o grande, al borde del propatagio con la abertura hacia el cuerpo (Figura 9C)

7. Antebrazo 40-45 mm; color gris pálido, más claro en el vientre, membranas café; cara y barbilla desnudas

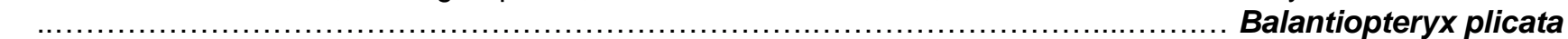

7'. Antebrazo 36-40 mm; color café oscuro, gris por debajo, membranas negras Balantiopteryx io No existe un registro oficial con espécimen de museo de esta especie en Honduras (Divoll y Buck 2013). 
8. Bolsa glandular en medio del propatagio (Figura 9), desde casi el borde hasta cerca del codo; antebrazo 46$48 \mathrm{~mm}$

Esta especie no ha sido reportada para Honduras pero existe en la Moskitia de Nicaragua muy cercano a la Moskitia hondureña (Reid 2009).

8'. Bolsa de pequeña a moderada. Se extiende del borde del propatagio hacia el codo, pero sin llegar cerca de este (Figura 9)

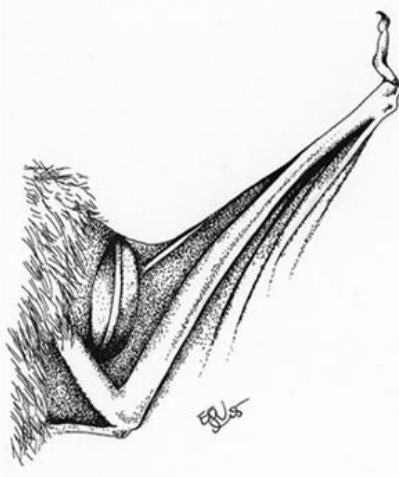

A

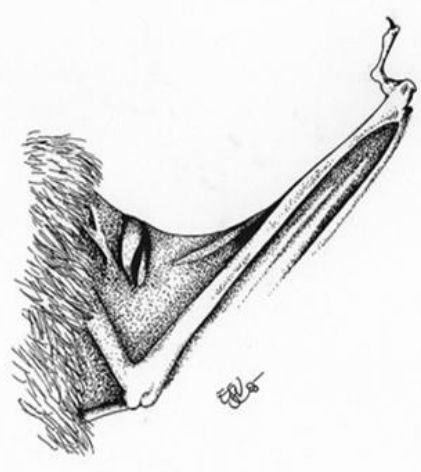

B

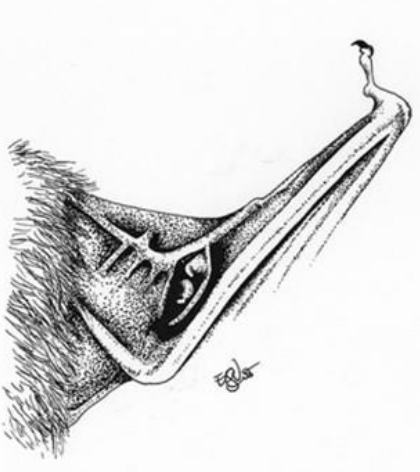

C

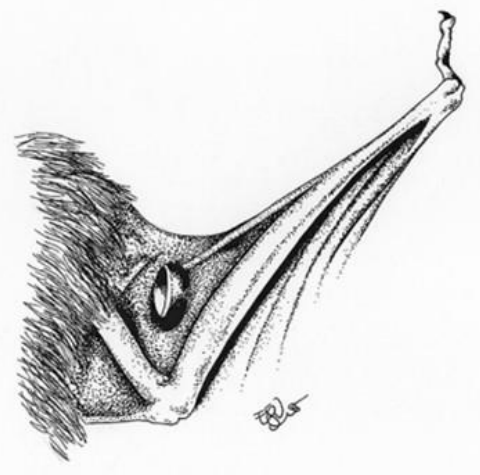

D

Figura 9. Sacos alares en el propatagio de A. Cormura brevirostris. B. Peropteryx spp. C. Saccopteryx spp, D. Balantiopteryx spp.

9. Pelaje café oscuro, de $8 \mathrm{~mm}$ en el cuello, $10 \mathrm{~mm}$ en espalda; antebrazo $45-50 \mathrm{~mm}$ (machos) y $46-52 \mathrm{~mm}$ (hembras).

Peropteryx kappleri

9'. Pelaje café rojizo, de $6 \mathrm{~mm}$ en el cuello, $8-9 \mathrm{~mm}$ en espalda; antebrazo $43-45 \mathrm{~mm}$ (machos) y $45-48 \mathrm{~mm}$ (hembras)

Peropteryx macrotis

\section{Clave para las Especies de Noctilionidae (Murciélagos Pescadores)}

1. Antebrazo más de $70 \mathrm{~mm}$; tibia y patas grandes, pata más de $25 \mathrm{~mm}$ de largo; garras alargadas; más de $50 \mathrm{~g}$ de peso

Noctilio leporinus

1'. Antebrazo menos de $70 \mathrm{~mm}$; pata menos de $25 \mathrm{~mm}$ de largo; garras no alargadas; menos de $50 \mathrm{~g}$

Noctilio albiventris

\section{Clave para las Especies de Mormoopidae (Murciélagos Bigotudos)}

1. Espalda de aspecto normal, con pelo, alas pegadas a los costados del cuerpo. 2

1 '. Espalda de aspecto desnuda, sin pelo, alas pegadas en el centro de la espalda

2. Orejas redondeadas, circundan los ojos y se conectan en la frente, unidas por un pliegue de piel con división en la parte alta; nariz hacia arriba; varios pliegues de piel en la barbilla (Figura 7); antebrazo 51-57 mm Mormoops megalophylla

2'. Labios gruesos, el inferior ensanchado, con un pliegue por debajo; con bigote largo a los lados; orejas angostas y puntiagudas (Figura 7); antebrazo $42-63 \mathrm{~mm}$ 3

3. Antebrazo menos de $50 \mathrm{~mm}$ Pteronotus personatus

3'. Antebrazo más de $50 \mathrm{~mm}$ Pteronotus mesoamericanus 


\section{Clave para las Subfamilias de Phyllostomidae}

1. Hoja nasal rudimentaria (Figura 3); incisivos superiores más anchos que los caninos (Figura 10); pulgar bien desarrollado Desmodontinae

1'. Hoja nasal prominente (Figura 3), con la excepción de Centurio senex (Figura 3); incisivos superiores menores que los caninos (Figura 10); pulgar pequeño ... 2

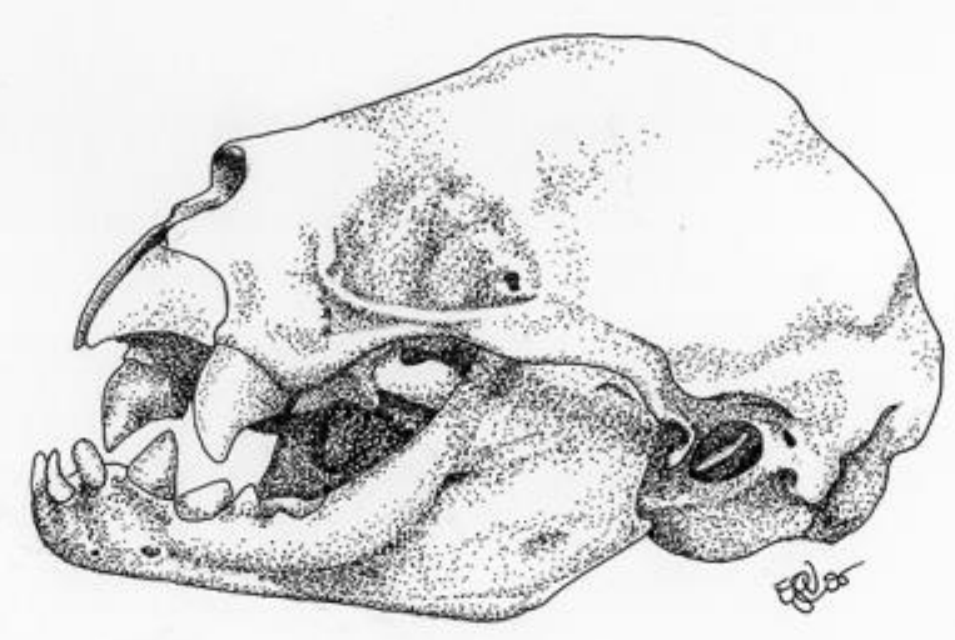

Figura 10. Incisivos superiores agrandados en Desmodontinae (cráneo de Desmodus rotundus).

2. Hocico largo y angosto; hoja nasal de forma de triángulo equilátero; orejas pequeñas y bien separadas; surco vertical profundo desde la boca hasta la barbilla; lengua larga

2'. Hocico ni largo ni angosto; hoja nasal generalmente larga; orejas generalmente largas; sin o con un surco pero no profundo en el labio inferior; lengua normal

3. Sin cola; uropatagio reducido y angosto; cabeza redondeada (Figura 3); hocico corto y redondeado; orejas no excepcionalmente grandes; hoja nasal bien desarrollada pero no excepcionalmente larga (con la excepción de Centurio senex, Figura 3); varias especies con líneas claras en la espalda o la cara ..... Stenodermatinae

3'. Con cola; uropatagio usualmente bien desarrollado; hocico no redondeado; orejas grandes o muy grandes; hoja nasal larga o muy larga

4. Barbilla con una verruga central rodeada de series de verrugas pequeñas y redondeadas en forma de $V$ (Figura 11); orejas y hoja nasal grandes; cola hasta la mitad del uropatagio donde sale a la superficie, aunque a veces apenas se nota; antebrazo $34-45 \mathrm{~mm}$; pelaje dorsal con bandas.

4'. Barbilla sin V de verrugas rodeando una verruga central agrandada; orejas y hoja nasal muy grandes; cola generalmente extendida dentro del uropatagio; antebrazo 31-116 mm

5. Hoja nasal y orejas muy largas, de longitud similar (Figura 12); antebrazo $49-54 \mathrm{~mm}$ Lonchorhininae 


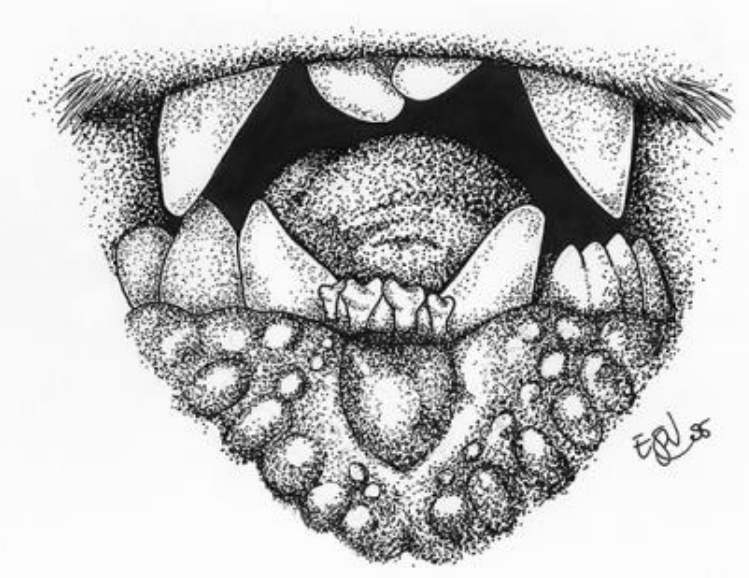

Figura 11. Barbilla con una verruga central rodeada de series de verrugas pequeñas y redondeadas en forma de $\checkmark$ en Carollinae.

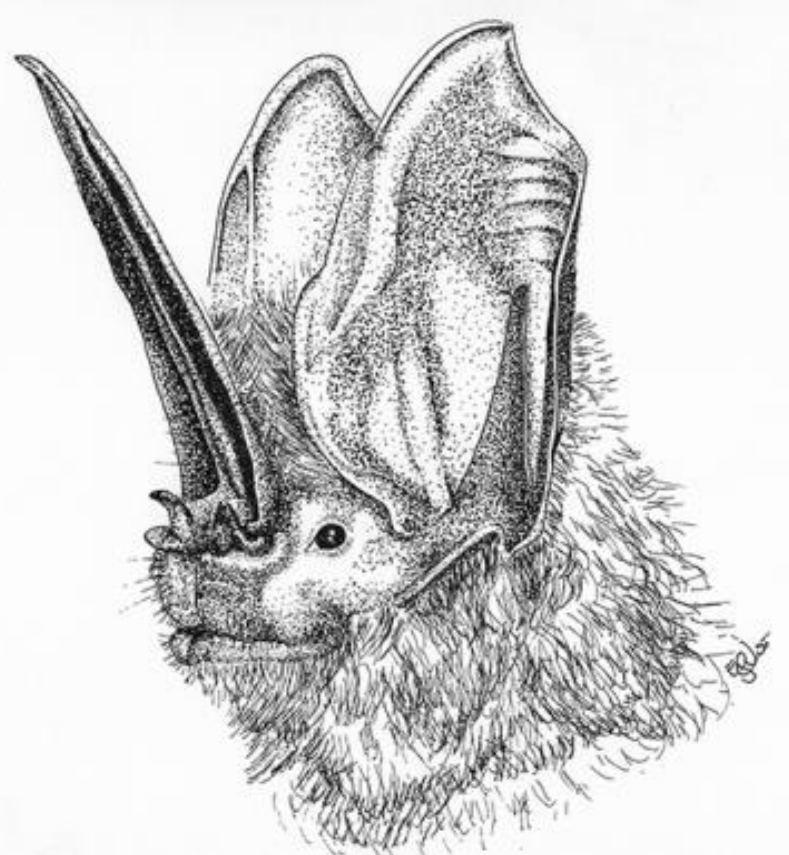

Figura 12. Esquema de Lonchorhina aurita (Lonchorhininae), donde se nota la longitud similar de las orejas y de la hoja nasal. Note las almohadillas en el labio inferior.

6. Sin dos almohadillas dérmicas con márgenes laterales redondeados y lisos en forma de $\mathrm{V}$ que bordea el surco de la barbilla Phyllostominae

6 '. Con dos almohadillas dérmicas con márgenes laterales redondeados y lisos en forma de $\mathrm{V}$ que bordea el surco de la barbilla 7

7. Reborde nasolabial engrosado con forma de cresta (Figura 13); uropatagio extenso; calcar igual o más largo que el pie

7'. Hoja nasal sin reborde nasolabial (Figura 13); uropatagio moderado, calcar más corto que el pie

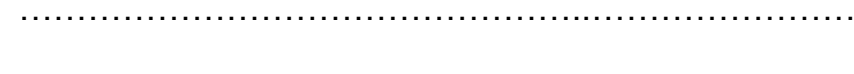

Glyphonycterinae 

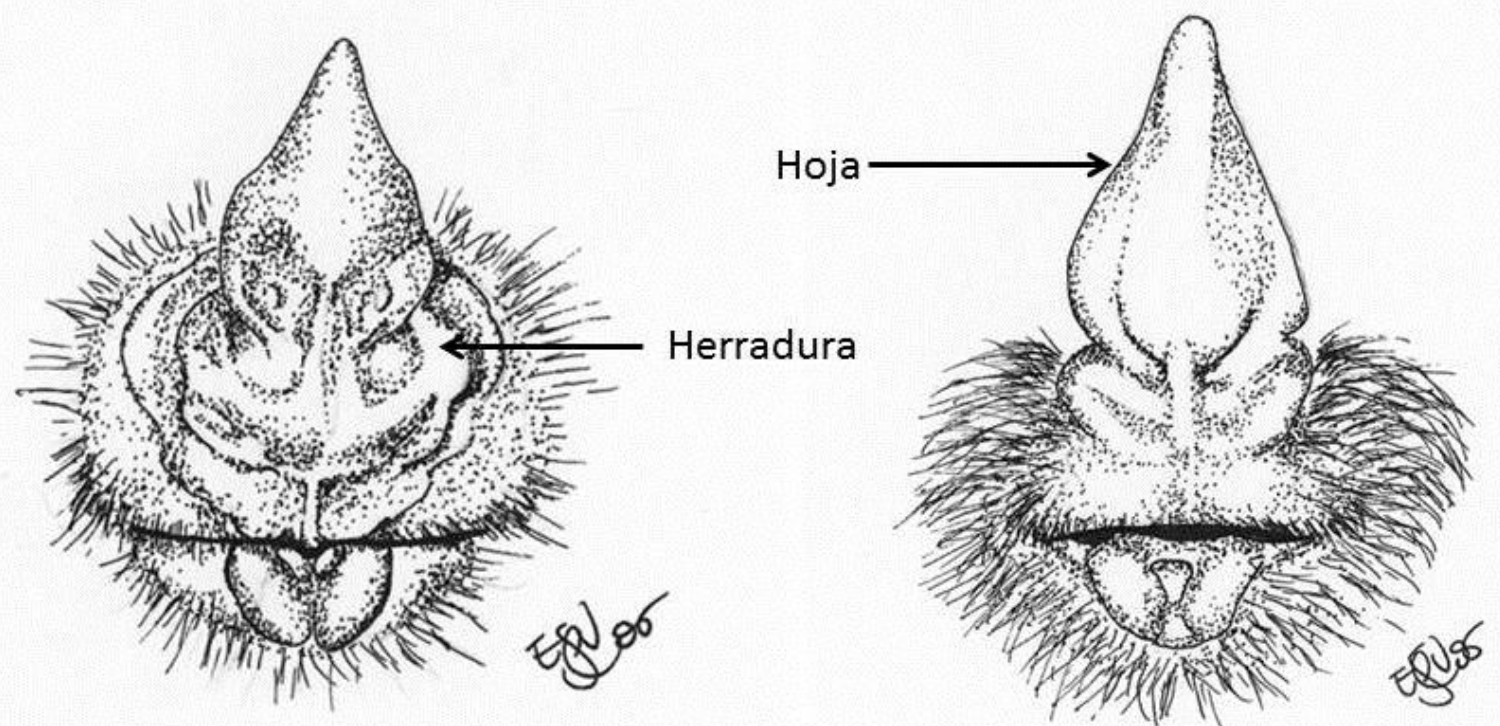

Figura 13. Hoja nasal de algunos murciélagos de la familia Phyllostomidae. A la izquierda una hoja nasal con reborde nasolabial pronunciado en forma de cresta en Lampronycteris brachyotis (Micronycterinae). A la derecha una hoja nasal sin reborde nasolabial en Trinycteris nicefori (Subfamilia Glyphonycterinae).

\section{Clave para las Especies de Micronycterinae (Murciélagos Recogedores)}

1. Pelo del vientre amarillo dorado o anaranjado a la base, amarillo brillante en la barbilla; antebrazo $38-43 \mathrm{~mm}$ Lampronycteris brachyotis

1'. Pelo del vientre café o blanco a la base 2

2. Antebrazo $40-46 \mathrm{~mm}$ Micronycteris hirsuta

2'. Antebrazo $32-38 \mathrm{~mm}$ 3

3. Pelaje ventral no más pálido que el dorsal; banda interauricular con un leve corte en $\mathrm{V}$; antebrazo 32-37 $\mathrm{mm}$. Micronycteris microtis

3'. Pelaje ventral pálido, gris o blanco- grisáceo; antebrazo 33-36 mm; banda interauricular con corte en $\mathrm{V}$ profundo 4

4. Calcar de $10 \mathrm{~mm}$, más largo que el pie; antebrazo $34-38 \mathrm{~mm}$ Micronycteris schmidtorum 4'. Calcar de 7-8 mm, más corto que el pie; antebrazo; 33-38 mm Micronycteris minuta

\section{Clave para las Especies de Glyphonycterinae (Murciélagos Recogedores)}

1. Antebrazo $54-58 \mathrm{~mm}$ Glyphonycteris daviesi

1'. Antebrazo $35-43 \mathrm{~mm}$ .2

2. Antebrazo $37-43 \mathrm{~mm}$; pelo dorsal con tres bandas (oscura, clara amplia, oscura); quinto metacarpo más largo Glyphonycteris sylvestris

2'. Antebrazo 37-40 mm; pelo dorsal con bandas pero tenues; 3er metacarpal más largo ...... Trinycteris nicefori La especie no ha sido reportada para Honduras, aunque existe en los países vecinos del norte y del sur. 


\section{Clave para las Especies de Desmodontinae (Vampiros)}

1. Ojos notoriamente grandes; orejas cortas con borde redondeado, uropatagio muy angosto y peludo, insertado en el centro del calcar; piernas muy peludas; pulgares sin cojinetes; antebrazo $54-56 \mathrm{~mm}$

Diphylla ecaudata

1'. Ojos no tan grandes; uropatagio angosto, sin o poco pelo; cojinetes en el pulgar; orejas con borde no redondeado; calcar vestigial o ausente

2. Alas de un solo color; pulgar largo con tres almohadillas evidentes (Figura 14); sin calcar; uropatagio reducido pero notorio; antebrazo 53-65 mm Desmodus rotundus

2'. Puntas de las alas blancas; pulgar largo con dos almohadillas poco evidentes (Figura 14); calcar vestigial; uropatagio muy reducido, antebrazo 50-56 m

Diaemus youngi

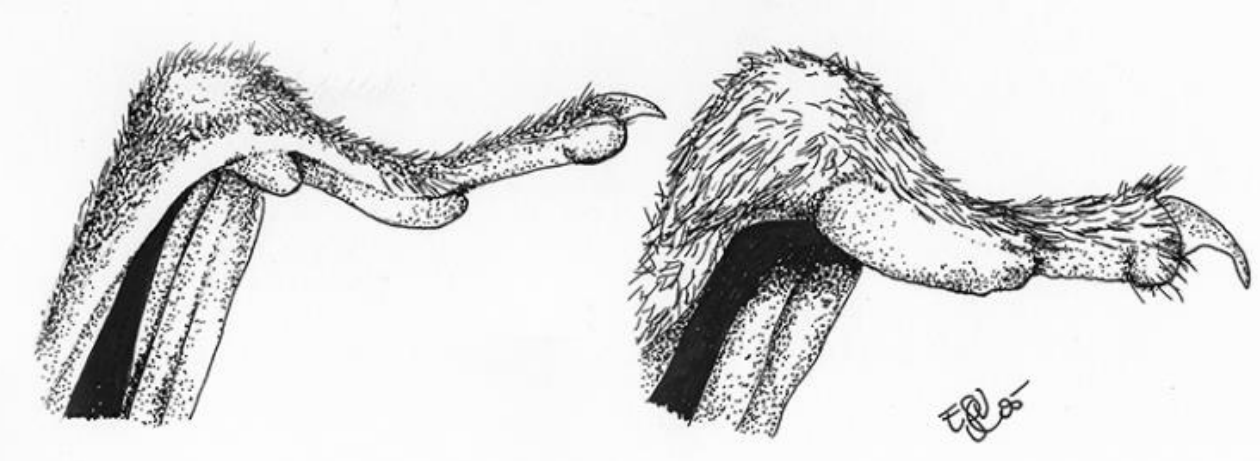

Figura 14. Pulgares de los desmodóntidos Desmodus rotundus (izquierda) y Diaemus youngi (derecha).

\section{Clave para las Especies de Lonchorhininae (Murciélagos Nariz de Espada)}

1* Antebrazo 49-54 mm; hoja nasal muy larga y angosta, largo similar al de las orejas (Figura 12); labio inferior con una hendidura central y una almohadilla a cada lado; cola y uropatagio muy largas *Solo hay una especie de Lonchorhininae en Honduras.

Lonchorhina aurita

\section{Clave para las Especies de Phyllostominae (Murciélagos Carnívoros y Recogedores)}

1. Antebrazo $69 \mathrm{~mm}$ o más.

1'. Antebrazo menos de $69 \mathrm{~mm}$

2. Sin cola o muy pequeña; orejas muy grandes y redondeadas .3

2'. Cola se extiende fuera del uropatagio; orejas moderadamente grandes, ligeramente puntiagudas ..... .4

3. Antebrazo $98-110 \mathrm{~mm}$

4. Puntas de las alas oscuras; antebrazo $80-93 \mathrm{~mm}$ 
5. Cola termina en el borde del uropatagio; área central interna del uropatagio con papilas dermales en líneas (Figura 15); antebrazo 34-37 mm

5'. Cola llega a la mitad del uropatagio como máximo 6

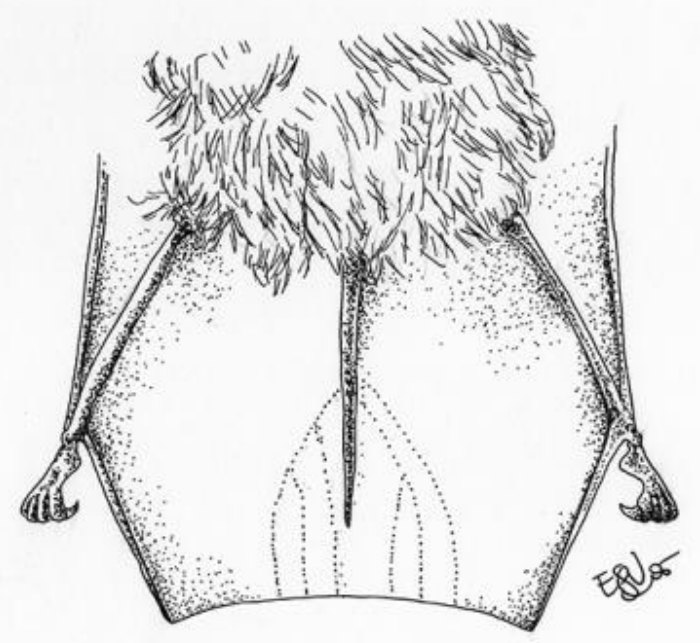

Figura 15. Uropatagio de Macrophyllum macrophyllum.

6. Labios y barbilla con "verrugas" alargadas alrededor de la boca (Figura 16); antebrazo 57-65 mm 6'. Sin verrugas en los labios, aunque puede tener en la barbilla; antebrazo $32-67 \mathrm{~mm}$
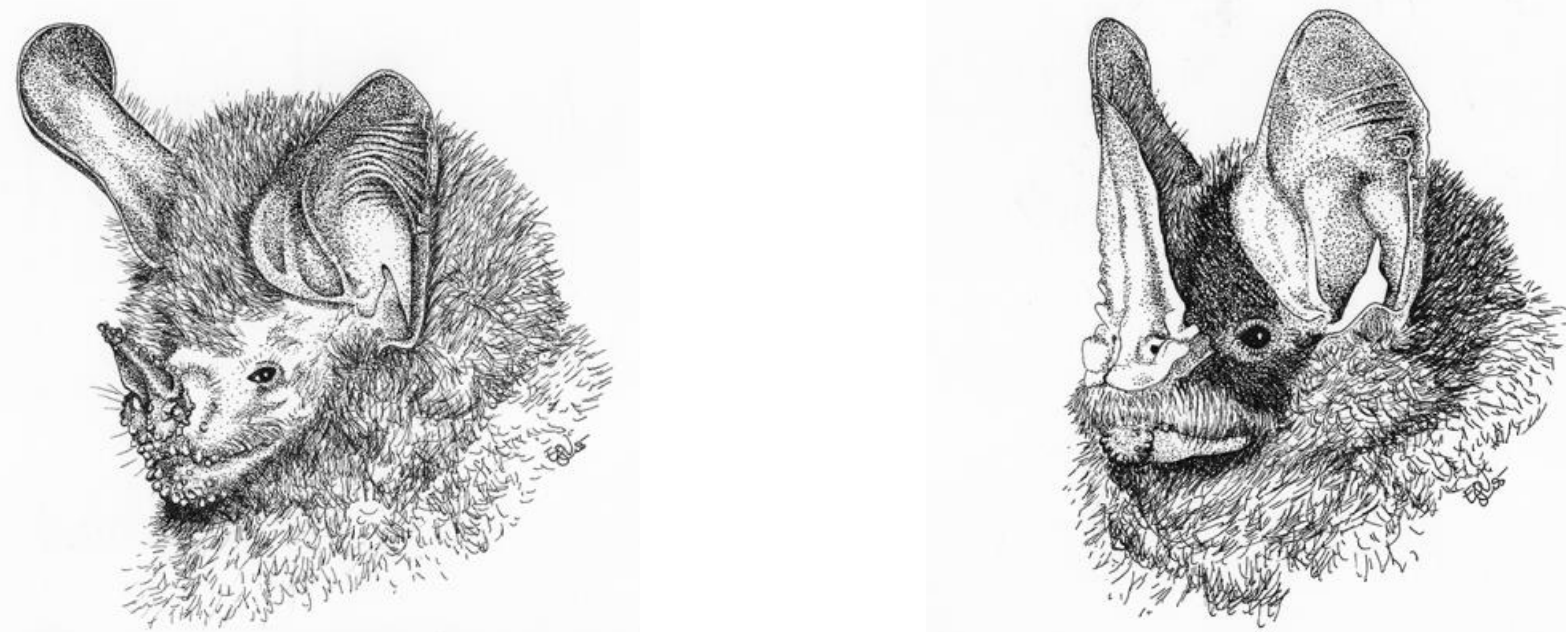

Figura 16. Dos filostóminos, Trachops cirrhosus (izquierda) y Gardnerycteris crenulatum (derecha).

7. Antebrazo 32-36 mm; protuberancias como verrugas agrupadas en forma de $\mathrm{V}$ en la punta de la barbilla

7'. Antebrazo 46-68 mm; punta de la barbilla variable según el caso Lophostoma brasiliense 
8. Orejas de tamaño medio, bien separadas entre sí; cola menos de un tercio del largo del fémur; antebrazo 60$68 \mathrm{~mm}$ Phyllostomus discolor

8'. Orejas grandes y cercanas entre sí; cola de longitud similar al fémur; antebrazo 46-61 mm 9

9. Hoja nasal larga y angosta (Figura 16, derecha); antebrazo 46-61 mm 10

9'. Hoja nasal en forma de triángulo equilátero; antebrazo 47-61mm 11

10. Antebrazo 46-55 mm; línea blanca en la espalda; con pelos en el borde externo de la hoja nasal

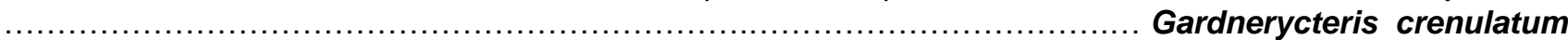

10'. Antebrazo 53-61 mm; sin línea blanca en la espalda; sin pelos en el borde externo de la hoja nasal Mimon cozumelae

11. Antebrazo 56-61 mm; raya pálida vertical de la coronilla a los ojos; base del antebrazo muy peluda; orejas no conectadas Tonatia saurophila

11'. Antebrazo 47-56 mm; sin raya clara en la coronilla; base del antebrazo sin pelo; sin raya blanca en la cabeza; orejas conectas por banda delgada 12

12. Generalmente con blanco en la garganta y en la base de las orejas, aunque algunos individuos no lo tienen; orejas 30-39 mm; antebrazo 50-56 mm; en Olancho y cercanías

Lophostoma silvicolum

12'. Generalmente sin blanco en la garganta ni en la base de las orejas, aunque algunos individuos lo tienen; muy similar al anterior, generalmente más pequeño; orejas 31-35 mm; antebrazo 47-54 mm; en la costa norte

Lophostoma evotis La coloración de estas dos especies varía y por lo tanto no es tan útil para diferenciarlas en el campo (Reid 2009).

\section{Clave de las Especies de Glossophaginae (Murciélagos Nectarívoros)}

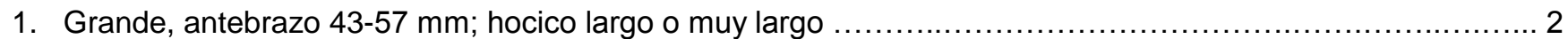

1'. Tamaño variable, antebrazo $30-45 \mathrm{~mm}$

2. Antebrazo $43-49 \mathrm{~mm}$; hocico muy largo y angosto; cola corta, se extiende $1 / 3$ del uropatagio, este sin pelos; patas no notoriamente peludas

Choeronycteris mexicana

2. Antebrazo 53-57 mm; sin cola, uropatagio corto en forma de $U$, con pelos en el borde; hoja nasal muy pequeña, triangular; pelaje blanco en la base Leptonycteris yerbabuenae

3. Con incisivos inferiores aunque no siempre evidentes .4

3. Sin incisivos inferiores

4. Incisivos internos superiores procumbentes, algo más largo que los externos; incisivos inferiores en contacto entre sí (Figura 17); base del pelo blancuzca; parte baja de la espalda a veces escarchada; antebrazo 33-38 $\mathrm{mm}$ Glossophaga soricina

4'. Incisivos superiores internos y externos aproximadamente del mismo tamaño (Figura 18), no procumbentes; incisivos inferiores usualmente con espacios notorios entre ellos 5

5. Antebrazo 32-35; incisivos inferiores muy reducidos, espaciados uniformemente (Figura 18); hoja nasal alargada; oscuro, nunca escarchado Glossophaga commissarisi

5'. Antebrazo 35-39 mm; incisivos inferiores reducidos, con espacios más grandes entre los incisivos interiores que entre los incisivos interiores y exteriores; hoja nasal no alargada

Glossophaga leachii

6. Antebrazo $40-45 \mathrm{~mm}$; sin cola; uropatagio reducido a una membrana estrecha muy peluda.. Anoura geoffroyi

6'. Antebrazo 31-35 mm ...7 
7. Ala unida a la base del dedo externo del pie (Figura 19); café oscuro; pelaje dorsal claramente tricolor, oscuro en la base; dos premolares y dos molares superiores; antebrazo $31-35 \mathrm{~mm}$

7'. Ala unida a la base del tobillo (Figura 19); dos premolares y tres molares superiores; antebrazo 31-35 mm

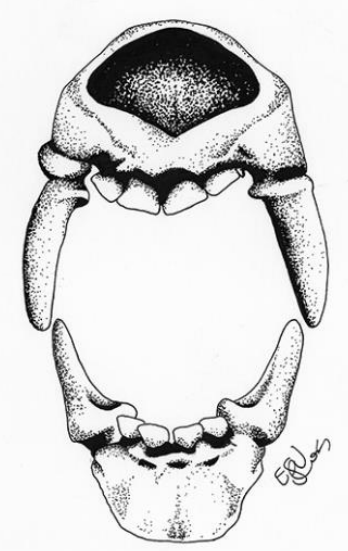

Figura 17. Caninos e incisivos superiores e inferiores de Glossophaga soricina.

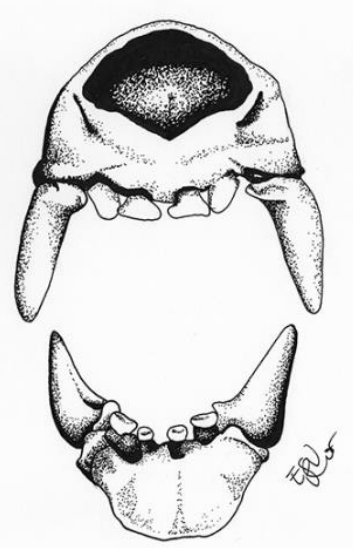

Figura 18. Caninos e incisivos superiores e inferiores de Glossophaga commissarisi.
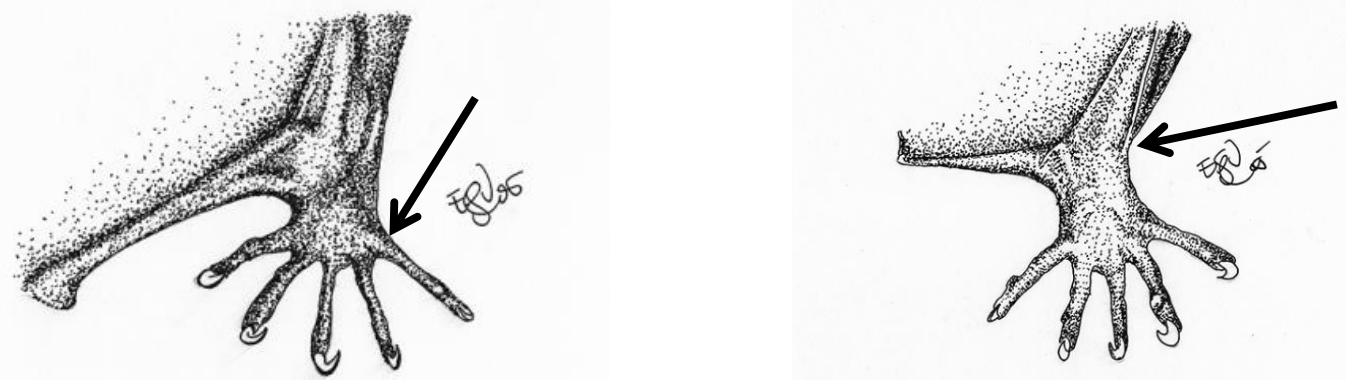

Figura 19. Ala unida a la base del dedo externo del pie (izquierda) y ala unida a la base del tobillo (derecha). 
8. Pelo de la espalda con dos bandas (claro-oscuro, Figura 20); incisivos superiores en dos pares separados por un espacio en medio; bigotes densos y largos; antebrazo 31-35 mm

Choeroniscus godmani

8'. Pelo de la espalda con tres bandas (oscuro-claro-oscuro, Figura 20); incisivos superiores separados de manera uniforme; antebrazo 31-34 mm Hylonycteris underwoodi
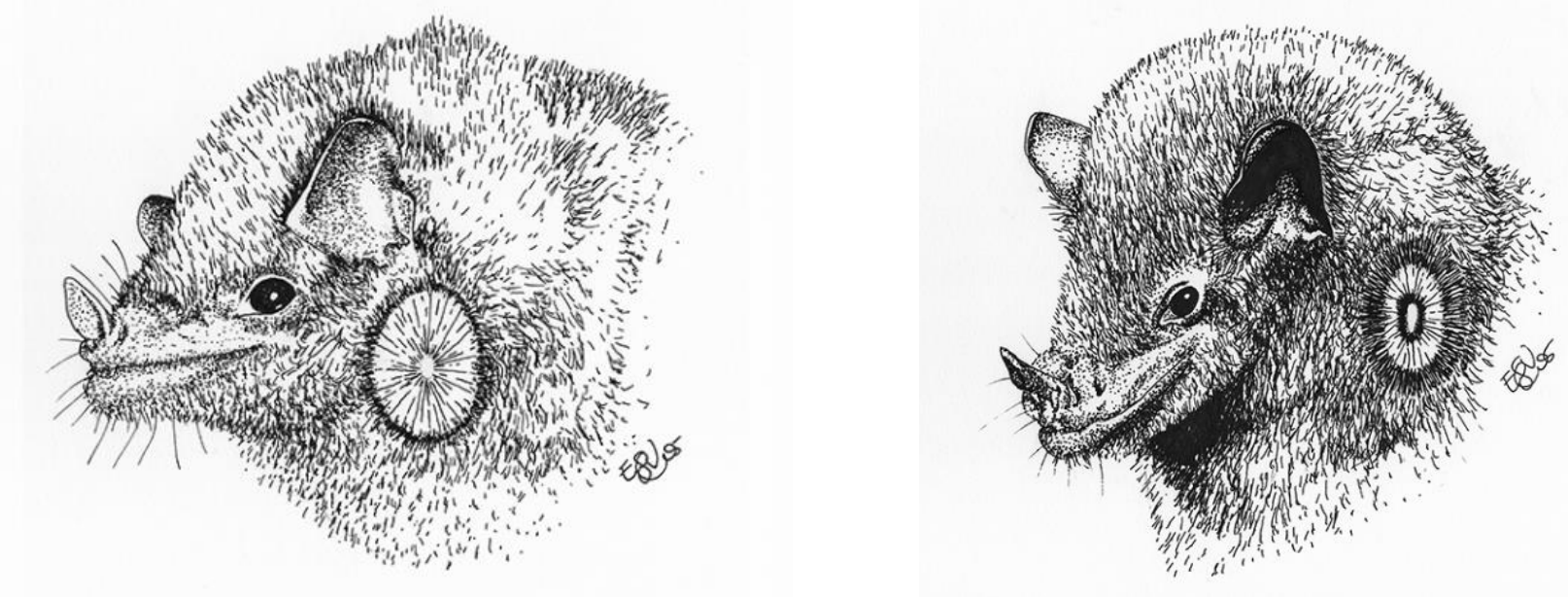

Figura 20. Pelo dorsal de Choeroniscus godmani con dos bandas (izquierda) y de Hylonycteris underwoodi con tres bandas (derecha).

\section{Clave de las Especies de Carollinae (Murciélagos Frugívoros de Cola Corta)}

1. Incisivos inferiores externos notablemente más cortos y anchos que los incisivos interiores (Ver figura 11); pelaje con tres bandas, 5-6 mm, banda central blancuzca de $2 \mathrm{~mm}$; antebrazo 41-45 mm; tibia 21-23 mm

Carollia perspicillata

1' Incisivos inferiores internos y externos similares en altura y forma, aunque los incisivos externos un poco más pequeños 2

2. Antebrazo y pies con abundante pelo; pelaje dorsal con tres bandas, $7-8 \mathrm{~mm}$, banda central blancuzca de $3 \mathrm{~mm}$; algunas veces levemente escarchado (lo que aparece como una cuarta banda del pelo); antebrazo 37$42 \mathrm{~mm}$; tibia 16-19 $\mathrm{mm}$ Carollia sowelli

2'. Antebrazo y pies con poco pelo; pelaje con tres bandas leve o claramente marcadas 3

3'. Pelaje castaño profundo, café rojizo o café apagado, ocasionalmente gris; tres bandas apenas notorias; antebrazo desnudo; antebrazo 34-38 mm; tibia 13.5-14.5 mm Carollia castanea

3. Pelaje con tres bandas, 6-7 mm, banda central blancuzca de 2-3 mm; algunas veces levemente escarchado (4 bandas); antebrazo $37-40 \mathrm{~mm}$; tibia $17-19 \mathrm{~mm}$

Carollia subrufa Carollia subrufa es muy similar a Carollia sowelli y sus ámbitos de distribución se sobreponen en Honduras. No obstante, $C$. sowelli tiene el antebrazo peludo, pelaje más largo con banda basal negruzca de 2-3 $\mathrm{mm}$, pero es de 1.0-1.5 en C. subrufa.

\section{Clave para las Especies de Stenodermatinae (Murciélagos Frugívoros)}

1. Uropatagio muy angosto y muy peludo 2

$1^{\prime}$. Urotapatagio notorio aunque puede ser reducido; extenso en varios casos 
2. Antebrazo 38-41 mm; incisivos inferiores trilobulados (Figura 21); incisivos superiores internos bicúspidos; pelaje rojizo; glándulas del hombro (charreteras) conspicuas; fémur y tibia con poco pelo; pelo corto (4.0-6.0 $\mathrm{mm}$ ) entre los hombros, bicolor

Sturnira parvidens

2'. Antebrazo 44-46 mm; incisivos inferiores bilobulados (Figura 21); incisivos superiores internos unicúspidos; color café oscuro o grisáceo; sin glándulas del hombro (charreteras); fémur y tibia peludas; pelo largo (7.0-10 $\mathrm{mm}$ ) entre los hombros, tetracolor

Sturnira hondurensis Las medidas del antebrazo de estas dos especies deben ser verificadas pues están basadas solo en algunos especímenes (Reid 2009, Velazco y Petterson 2013).
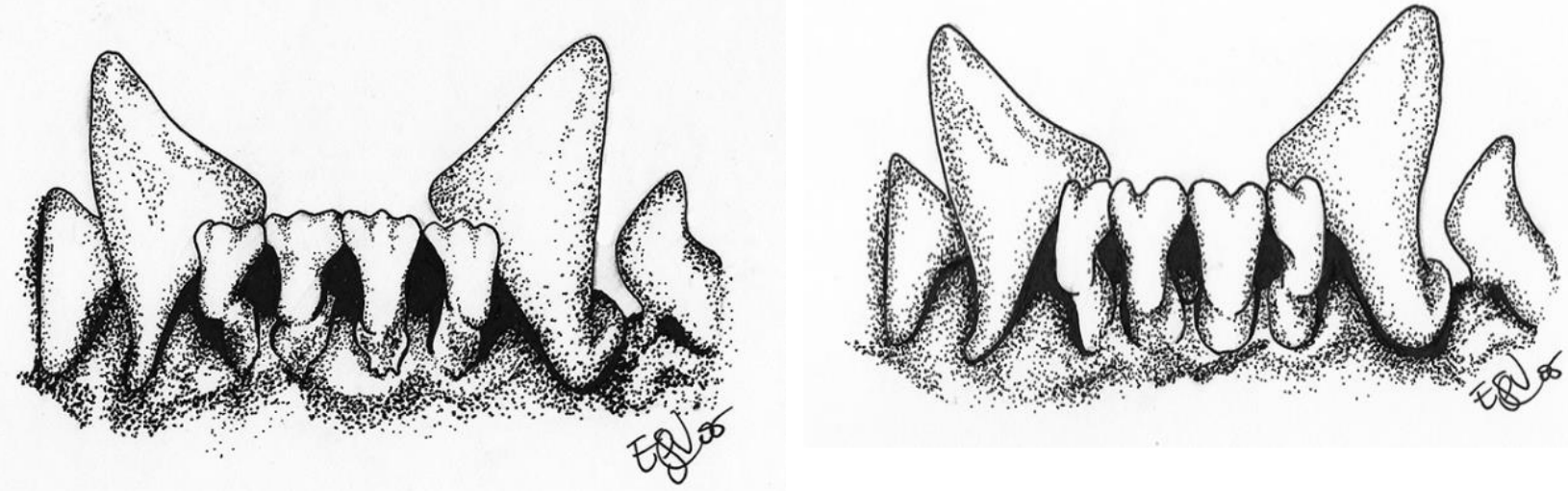

Figura 21. Incisivos inferiores trilobulados en Sturnira parvidens (izquierda) y bilobulados en Sturnira hondurensis (derecha).

3. Inconfundible, cara desnuda con pliegues y arrugas, sin hoja nasal (Figura 3); una mancha blanca en cada hombro (charreteras) con un patrón entramado de líneas de piel pigmentada y transparente; antebrazo 41-45 $\mathrm{mm}$

Centurio senex

3'. Cara con apariencia normal, peluda, sin pliegues ni arrugas, con hoja nasal; sin mancha blanca en el hombro; sin patrón diferenciado en las alas

4

4. Color blanco o blanco grisáceo; antebrazo 25-30 mm; membranas negras; orejas y hoja nasal de tonalidad amarilla o anaranjado

Ectophylla alba

4'. Color oscuro; antebrazo variable 5

5. Vientre con pelo con apariencia de puntas blancas lo que le da una tonalidad escarchada 6

$5^{\prime}$. Vientre no escarchado como el anterior 7

6. Antebrazo 55-76; mitad basal de la superficie dorsal del uropatagio sin pelaje; con líneas faciales, a veces poco evidentes

Artibeus jamaicensis

6'. Antebrazo 48-53 mm; pelo corto y aterciopelado; línea facial inferior a veces ausente; uropatagio con pelos dispersos, borde con pelos en el centro Artibeus inopinatus

7. Línea blanca en la espalda 14

7'. Sin línea blanca en la espalda

8. Antebrazo más de $60 \mathrm{~mm}$; líneas faciales de poco evidentes a muy marcadas Artibeus lituratus

8'. Antebrazo menos de $55 \mathrm{~mm}$; líneas faciales variables

9. Antebrazo 30-32 mm; color café pálido; líneas faciales poco evidentes; uropatagio con orla de pelo en el borde

$9^{\prime}$. Antebrazo 35-50 mm; color variable; líneas faciales variables Vampyressa thyone 
10. Antebrazo $38-42 \mathrm{~mm}$; incisivos superiores internos cortos, anchos, planos; líneas faciales marcadas de color anteado; uropatagio angosto con orla de pelos

10’. Incisivos superiores de forma similar, los centrales con dos lóbulos, el externo un poco más pequeño 11

11. Líneas de la cara bien marcadas; café o gris; uropatagio 9-12 mm casi desnudo y sin orla de pelos en el borde

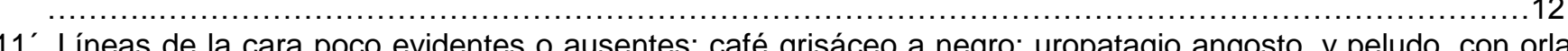
de pelos en el borde 13

12. Pelo de 4-6 $\mathrm{mm}$; uropatagio de $9-11 \mathrm{~mm}$; orejas con bordes amarillentos o crema aunque algunas veces puede ser blanco; antebrazo $35-42 \mathrm{~mm}$

Artibeus phaeotis

12'. Pelo de 6-7 mm; uropatagio de 10-12 mm; orejas con bordes blancuzcos aunque algunas veces puede ser amarillo; antebrazo 35-41 $\mathrm{mm}$ Artibeus watsoni

13. Pelaje café carbón a negruzco; pelo $9-10 \mathrm{~mm}$; uropatagio $6 \mathrm{~mm}$, en forma de $\mathrm{V}$, superficie dorsal peluda y orla densa (de $5 \mathrm{~mm}$ ); antebrazo 41-49 $\mathrm{mm}$

Artibeus aztecus

13'. Pelaje café grisáceo, a veces negruzco; pelo 6-8 $\mathrm{mm}$ ); uropatagio 8-9 $\mathrm{mm}$, en forma de $U$, superficie dorsal ligeramente peluda, orla rala (de $3 \mathrm{~mm}$ ); antebrazo $39-43 \mathrm{~mm}$

Artibeus toltecus

14'. Líneas en la espalda poco evidentes; incisivos superiores internos simples o bilobulados, más del doble del largo de los incisivos externos

14. Líneas en la espalda bien marcadas 16

15. Antebrazo $42-47 \mathrm{~mm}$; línea dorsal a veces ausente; incisivos superiores internos angostos, muchos más largos que los externos (Figura 22); líneas faciales no evidentes o ausentes; pelo tricolor; pelos guarda largos prominentes dispersos entre el pelaje; ojos grandes; uropatagio largo $(19 \mathrm{~mm})$, peludo por encima, sin orla de pelos en el borde Chiroderma villosum

15'. Antebrazo 35-39 mm; líneas faciales marcadas; incisivos superiores internos con dos lóbulos (el interno más largo), muy diferentes en tamaño y forma de los incisivos externos (Figura 23); orejas amarillas; hoja nasal clara

Vampyriscus nymphaea

16. Antebrazo $47 \mathrm{~mm}$ o más 17

16'. Antebrazo $47 \mathrm{~mm}$ o menos 18

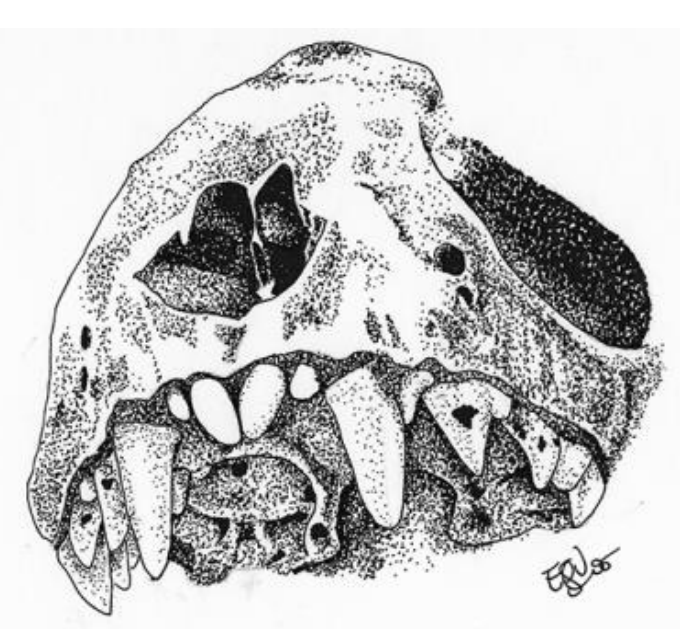

Figura 22. Cráneo de un ejemplar de Chiroderma sp. Nótese la longitud de los incisivos centrales en relación a los externos. 


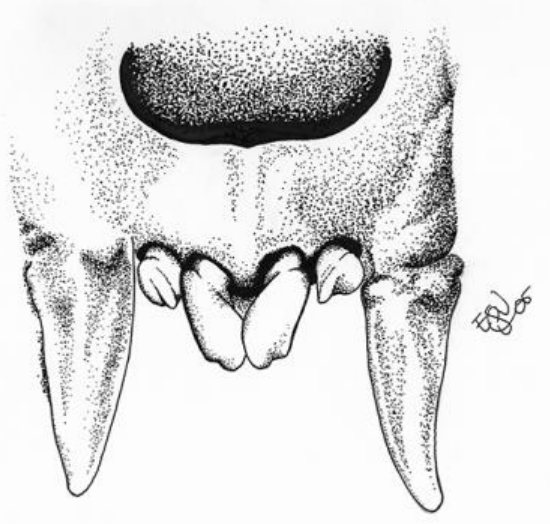

Figura 23. Caninos e incisivos superiores de Vampyriscus nymphaea.

17. Café pálido o rojizo; pelo bicolor, pálido en la base; línea dorsal desde la coronilla hasta las ancas; líneas faciales bien marcadas, la superior hasta atrás de las orejas; uropatagio corto $(10 \mathrm{~mm})$, con orla de pelos claros; antebrazo 47-56 mm

Vampyrodes major

17'. Café claro a oscuro; líneas faciales bien marcadas, la superior hasta las orejas; pelo dorsal con tres bandas, oscuro en la base; línea dorsal desde los hombros hasta las ancas, a veces poco evidente; rostro aplanado; uropatagio notorio $(15 \mathrm{~mm})$, con pelos en la superficie pero sin orla notoria; antebrazo 48-52

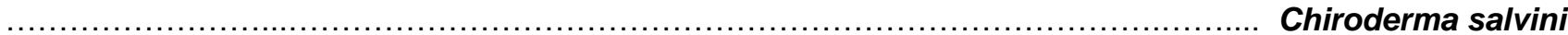

18. Rayas faciales marcadas; orejas bordeadas de amarillo o blanco ........................................... 19

18'. Rayas faciales poco evidentes; orejas sin bordes amarillo o blanco; antebrazo 41-45 mm Uroderma magnirostrum

19. Uropatagio muy angosto, borde muy peludo; línea dorsal comienza al nivel de las orejas; antebrazo 37-41 $\mathrm{mm}$.

Platyrrhinus helleri

19'. Uropatagio notorio, sin pelos en el borde; línea dorsal comienza en la parte superior de la espalda; incisivos superiores internos con dos lóbulos (Figura 24), más grandes que los incisivos externos; antebrazo 40-44 $\mathrm{mm}$

20. Principalmente en el occidente del país

Uroderma davisi

$20^{\prime}$. En el resto del país

Uroderma convexum

Estas dos especies son prácticamente idénticas por lo que son imposibles de distinguir en el campo. Tienen diferencias craneales; $U$. davisi es $2 \mathrm{~N}=44$ y $U$. convexum es $2 \mathrm{~N}=38$ (Mantilla-Meluk 2014).

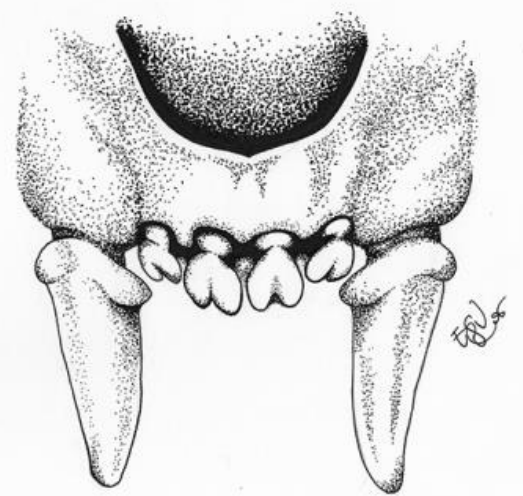

Figura 24. Caninos e incisivos superiores en Uroderma spp. 


\section{Clave para las Especies de Thyropteridae (Murciélagos Platanilleros)}

$1^{*}$ Antebrazo 35-38 mm; cola se extiende 5-8 mm fuera de la membrana de la cola; uropatagio casi desnudo.

Thyroptera tricolor *Solo hay una especie de Thyropteridae en Honduras (Figura 2).

\section{Clave Para las Especies de Natalidae (Murciélagos Orejas de Embudo)}

1. Pelo ventral de color uniforme, base de las garras con pelos cortos, sin pelos pequeños erectos en la tibia, tobillo y calcar (Figura 25); antebrazo 35-37 mm Natalus mexicanus

$1^{\prime}$. Pelo ventral bicolor, base de las garras con pelos largos, pelos pequeños erectos en la tibia, tobillo y calcar (Figura 25); antebrazo 35-39 mm Natalus lanatus
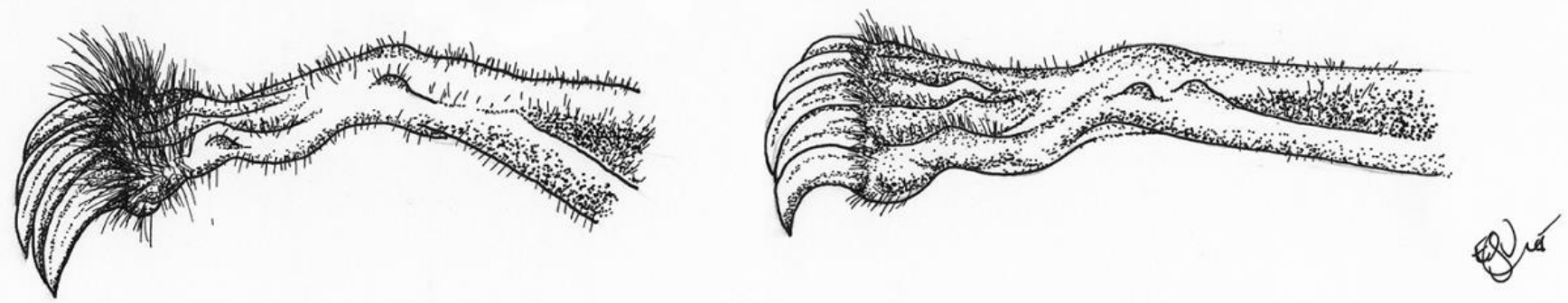

Figura 25. Base de las garras con pelos largos, pelos pequeños erectos en la tibia, tobillo y calcar en Natalus lanatus en comparación a Natalus mexicanus (derecha).

\section{Clave para las Especies de Vespertilionidae (Murciélagos Vespertinos)}

1. Orejas largas, al extenderse hacia adelante se proyectan más allá del hocico; hocico truncado similar al cerdo; ventanas de la nariz abiertas hacia adelante, con pliegue de forma espiralada (Figura 26); antebrazo 49-56 $\mathrm{mm}$

Bauerus dubiaquercus

1'. Orejas comparativamente cortas, al extenderse hacia adelante no se proyectan más allá del hocico; ventanas de la nariz abiertas hacia los lados o hacia adelante pero sin pliegue espiralado

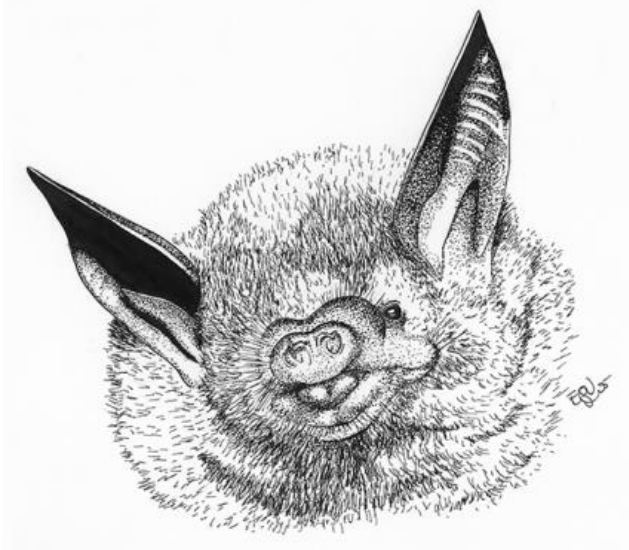

Figura 26. Rostro de Bauerus dubiaquercus, nótese el pliegue en forma de espiral en la nariz. 
Mora: Clave para la Identificación de las Especies de Murciélagos de Honduras

2. Orejas de largo moderado, más o menos puntiagudas; superficie del uropatagio con poco pelo o solo en la

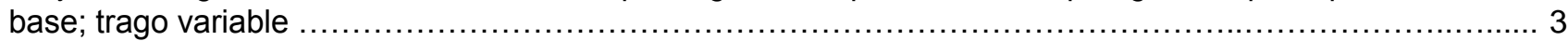

2 '. Orejas cortas y redondeadas; superficie dorsal del uropatagio con pelo por lo menos hasta la mitad; trago de forma triangular o redondeado ...4

3. Pelo más oscuro en la base que en la punta; trago curvado hacia adelante, ancho y con punta redonda; dos premolares, uno muy pequeño y el otro más grande entre el pequeño y el canino (1/3 de este); antebrazo 31 $35 \mathrm{~mm}$

Perimyotis subflavus

3'. Pelo más oscuro en la base que en la punta o del mismo color; trago recto angosto y punteado; tres premolares, solo uno grande, con o sin diastema aparente 8

4. Antebrazo 43-58 mm; espalda anteada, amarillenta, café amarillento o caoba; superficie dorsal del uropatagio cubierta de pelo 5

4'. Antebrazo 38-50 mm; espalda naranja rojiza a café rojizo o color rojo anaranjado brillante; superficie dorsal del uropatagio cubierta de pelo .7

5. Inconfundible, espalda café amarillento a caoba, muy escarchado con plateado, banda amarillenta alrededor de la cara y garganta; todo el uropatagio con pelaje denso similar al del dorso; antebrazo 50-57 mm

Lasiurus cinereus

5'. Pelo largo y suave, amarillo opaco, anteado grisáceo; pelo amarillo brillante o naranja pálido en el uropatagio; patas y mitad distal del uropatagio sin pelo 6

6. Antebrazo $43-47 \mathrm{~mm}$ Lasiurus ega

6'. Antebrazo $48-58 \mathrm{~mm}$ Lasiurus intermedius

7. Antebrazo 38-42 $\mathrm{mm}$; rojizo o café rojizo; piernas y uropatagio muy peludas Lasiurus frantzii

7'. Antebrazo 49-50 mm; rojo anaranjado brillante, más oscuro en el uropatagio; pelo de la cabeza y el cuello con una banda con una sección crema notoria; vientre naranja brillante Lasiurus egregius Esta especie es extremadamente rara y ha sido reportada para Honduras en una sola ocasión (Mora 2012).

8. Coloración café amarillenta, pelos con base amarilla y punta oscura; vientre café claro a oscuro; dos incisivos superiores; antebrazo $29-31 \mathrm{~mm}$ 9

8'. Coloración dorsal varía de rojizo a café a casi negro; coloración ventral similar; cuatro incisivos superiores; antebrazo $31-54 \mathrm{~mm}$ 10

9. Punta del pelo bicolor, de oscura a café claro con base amarilla anteada; pelo ventral varía de café claro a oscuro; base del uropatagio con pelo; orejas cortas (10-12 mm), oscuras La localidad tipo de Rhogeessa menchuae es Lancetilla, Atlántida. Rhogeessa menchuae

9'. Punta del pelo de color marrón o negro intenso, con base generalmente gris a amarillo anteado; pelo ventral marrón claro; sin franja de pelo en el uropatagio; orejas cortas (11-14 mm), oscuras...Rhogheessa bickhami En Comayagua y Valle.

Estas dos especies son casi idénticas, por lo que es casi imposible distinguirlas en el campo. Incluso, las especies no tienen diferencias en el cariotipo. $R$. menchuae tiene un pelaje y orejas más claras, proyección angular más elevada, rostro más corto, incisivos superiores más grandes y más delgado y M3 más pequeño.

10. Narinas laterales; un premolar superior grande y conspicuo evidentemente más largo que los molares, en contacto con el canino (Figura 27); incisivos superiores internos anchos bilobulados; incisivos externos poco notorios; trago chato y curvado hacia adelante; antebrazo $37-54 \mathrm{~mm}$ 11

10'. Narinas frontales; tres premolares superiores, uno grande, separado del canino por dos muy pequeños aparentando una diastema (Figura 27); incisivos superiores interiores con apariencia cónica; incisivos exteriores de longitud similar; trago recto, angosto y punteado; antebrazo $31-44 \mathrm{~mm}$ 13 
11. Antebrazo 37-41 mm; negruzco o café oscuro, a veces escarchado, vientre anteado; pelaje 5-6 mm; piel de la cara café rosado, membranas negruzcas

11'. Antebrazo 39-52 mm; café naranja, amarillento, café, café negruzco o negruzco; pelaje 6-10 mm ............ 12

12. Antebrazo 46-52 mm; café naranja, amarillento o café, vientre anteado, base del pelo negra; pelaje 8-10 mm; piel de la cara, orejas y membranas negras

Eptesicus fuscus

12'. Antebrazo 39-43 mm; café naranja a negruzco; vientre anteado-café; pelaje 6-7 mm; piel de la cara café rosado alrededor de los ojos, hocico oscuro, membranas oscuras

Eptesicus brasiliensis Generalmente en tierras más altas que E. furinalis que se encuentra desde las tierras bajas hasta los 1,800 msnm, usualmente debajo de $500 \mathrm{msnm}$, E. brasiliensis desde tierras bajas a los 3,000 msnm (Reid 2009).
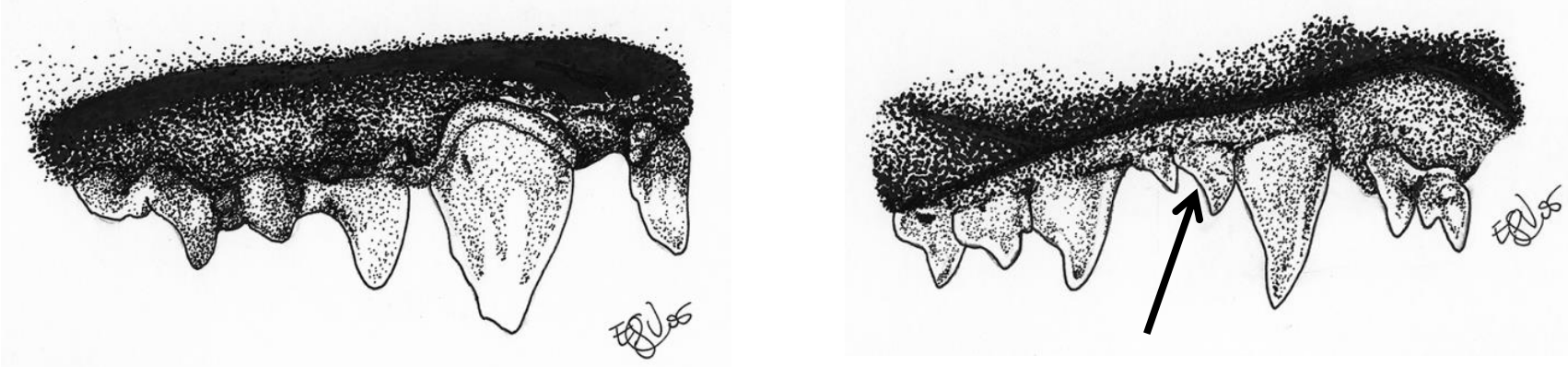

Figura 27. Premolar superior en contacto con el canino superior en Eptesiscus (izquierda). Premolar grande separado del canino por dos pequeños (señalados) en Myotis (derecha).

13. Uropatagio con pelo hasta la mitad entre la rodilla y el pie o alcanza el pie; gris rosáceo a café grisáceo oscuro; antebrazo 32-39 mm Myotis keaysi

13'. Uropatagio con pelo hasta la rodilla o menos; generalmente grisáceo o negruzco 14

14. Pelaje dorsal negruzco o negro, notoriamente escarchado; vientre gris, palidece hacia casi blanco en el vientre inferior y alrededor de las piernas; antebrazo $33-38 \mathrm{~mm}$ Myotis albescens

14'. Pelaje dorsal de color variable pero no escarchado 15

15. Antebrazo 41-47 mm; pelaje como lana, café grisáceo, a veces con parche vacío entre los hombros; poco contraste entre las puntas y las bases

Myotis velifer

15. Antebrazo 31-39 $\mathrm{mm}$; pelaje variable, pero raramente como lana. 16

16. Antebrazo 33-38 mm; coloración oscura, casi negro; pelaje sedoso; 1er y 2 do premolares superiores pequeños no apiñados en la fila dental, 2 do premolar ligeramente más pequeño que el primero; 2do incisivo inferior similar al primero; incisivo 1 angosto

Myotis nigricans

16'. Antebrazo 32-39 mm; coloración grisácea, parduzca o café rojizo; 1er y 2 do premolares superiores pequeños apiñados en la fila dental, 2do premolar claramente más pequeño que el primero; incisivo 1 ancho .17

17. Antebrazo $32-35 \mathrm{~mm}$; de café pálido a rojizo; pelaje sedoso, con dos bandas; premolares superiores pequeños apiñados, pero alineados en la fila; 2do incisivo inferior similar al primero

Myotis elegans

17'. Antebrazo 32-39 mm; de gris oscuro a café grisáceo; pelaje como lana de un solo color; 2do premolar superior apiñado adentro de la fila por lo que de lado no se ve; 2do incisivo ligeramente más alto que el incisivo 1.

Myotis riparius

Las especies de Myotis son sumamente difíciles de identificar con seguridad en el campo. Es recomendable la manipulación amable de los animales y evitar recolectas innecesarias. Además, es posible que en realidad algunas de estas especies no sean las que se cree que son. 


\section{Clave para las Especies de Molossidae (Murciélagos de Cola Libre)}

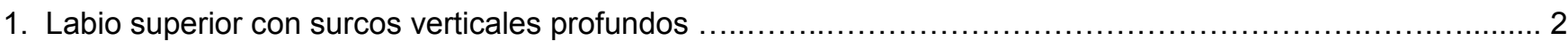

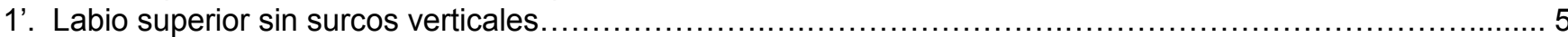

2. Orejas juntas pero no unidas en la base, cuando se doblan hacia adelante no se proyectan más allá del hocico; 2do falange del 4to dedo más de $5 \mathrm{~mm}$; antebrazo $37-46 \mathrm{~mm}$

Tadarida brasilensis

2'. Orejas unidas en la base, cuando se doblan hacia adelante se proyectan más allá del hocico; 2do falange del 4to dedo menos de $5 \mathrm{~mm}$

3. Antebrazo $41-45 \mathrm{~mm}$

Nyctinomops laticaudatus

3. Antebrazo mayor a $45 \mathrm{~mm}$

4. Antebrazo menos de $55 \mathrm{~mm}$

Nyctinomops aurispinosus

4'. Antebrazo más de $55 \mathrm{~mm}$ Nyctinomops macrotis

5. Orejas usualmente unidas en la base, cuando se doblan hacia adelante se proyectan más allá del hocico (Figura 28); antitrago no circular 6

5'. Orejas unidas en la base, cuando se doblan hacia adelante no se proyectan más allá del hocico (Figura 28); antitrago circular, grande 10

6. Antebrazo menos de $52 \mathrm{~mm}$ 7

6'. Antebrazo más de $52 \mathrm{~mm}$ 8
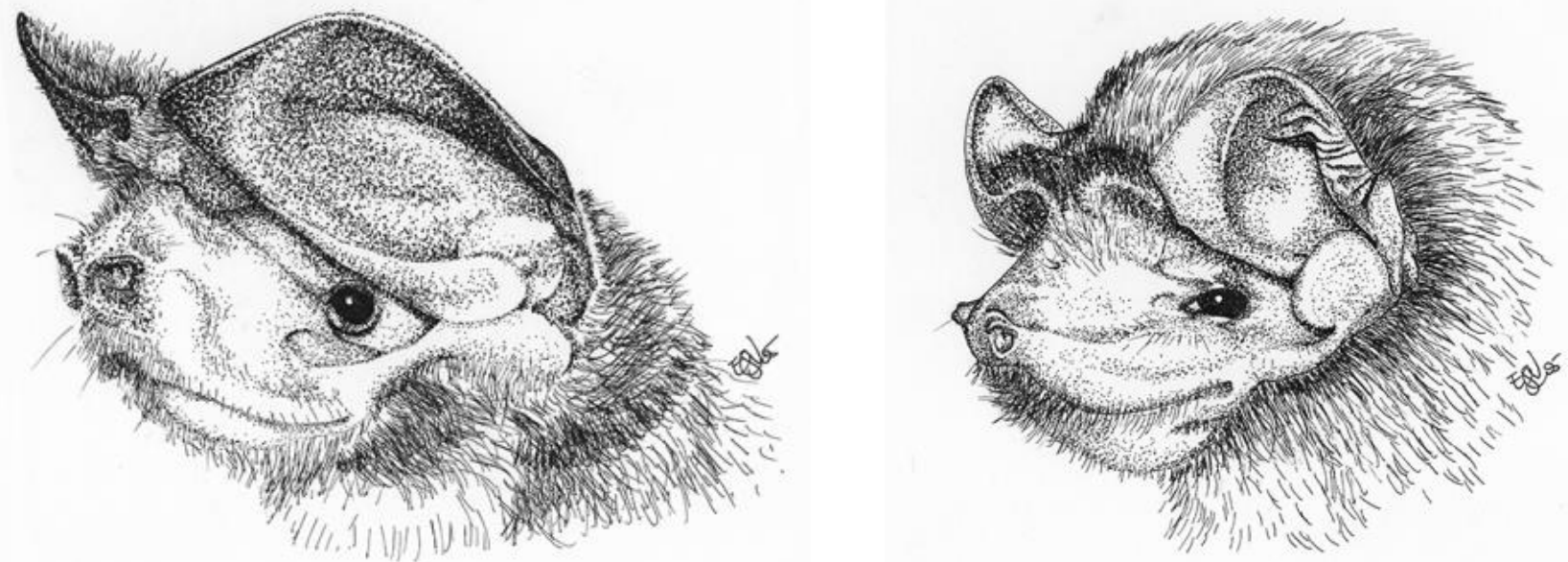

Figura 28. Orejas de molósidos, grandes en Eumops (izquierda), pequeñas en Molossus (derecha).

7. Dorso café grisáceo o café, más pálido en la base, de $5 \mathrm{~mm}$; con algunos pelos cortos en el borde del calcar; sin pelos largos en la rabadilla; antebrazo $39-48 \mathrm{~mm}$

Eumops nanus

7'. Dorso café oscuro o negruzco, oscuro a la base, de $2-3 \mathrm{~mm}$; sin pelos en el borde del calcar; con pelos largos en la rabadilla $(8 \mathrm{~mm})$; antebrazo $37-42 \mathrm{~mm}$

Eumops hansae

8. Antebrazo $57-63 \mathrm{~mm}$; pelo de color negro o muy oscuro hasta la base; sin pelos largos en la rabadilla

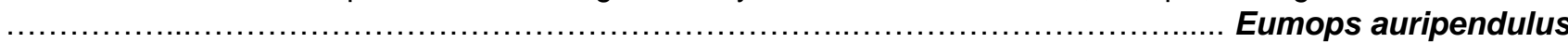

8'. Antebrazo 55-74 mm; pelo de color claro o moderadamente oscuro, más pálido en la base; pelos largos en la rabadilla 
9. Antebrazo $55-63 \mathrm{~mm}$; pelo café grisáceo

Eumops glaucinus

9'. Antebrazo $66-74 \mathrm{~mm}$; pelo gris o café rojizo Eumops underwoodi

10. Cuatro incisivos inferiores 11

10'. Dos incisivos inferiores 12

11. Antebrazo $34-38 \mathrm{~mm}$

Cynomops mexicanus

11'. Antebrazo $51-57 \mathrm{~mm}$ Promops centralis

12. Antebrazo más de $44 \mathrm{~mm}$...... 13

12'. Antebrazo menos de $44 \mathrm{~mm}$ .14

13. Negro, café oscuro o café rojizo, base del pelo blanca; cerdas en las ancas de 11-12 mm; antebrazo 45-52 $\mathrm{mm}$

Molossus sinaloae

13'. Negro, café chocolate oscuro o anaranjado oscuro, base del pelo oscura, cerdas en las ancas de 8-9 mm; antebrazo $47-54 \mathrm{~mm}$ Molossus rufus

14. Base del pelo negra; pelo de $2 \mathrm{~mm}$, negro a la base; antebrazo $38-43 \mathrm{~mm}$ Molossus bondae

14'. Base del pelo blanca; pelo de 2-4 $\mathrm{mm}$; antebrazo $33-40 \mathrm{~mm}$ 15

15. Base del pelo blanca; café grisáceo pálido a café oscuro; rostro y orejas café; antebrazo 36-40 mm; cerdas de 8-10 $\mathrm{mm}$ (no siempre) Molossus molossus

$15^{\prime}$. Base del pelo blanca por un cuarto de la longitud de este; antebrazo $33-38 \mathrm{~mm}$ 16

16. Café oscuro o negro; pelo 2-3 mm cerca del hombro; cerdas en las ancas $8-10 \mathrm{~mm}$; antebrazo $33-37$

16'. Color café chocolate oscuro o negruzco; pelaje $4 \mathrm{~mm}$ cerca del hombro; antebrazo $36-38 \mathrm{~mm}$ Molossus aztecus No está claro si esta especie existe en Honduras, pero su distribución indica que debe estar presente en el país (Reid 2009). Estas tres últimas especies son muy difíciles de diferenciar en el campo (Reid 2009).

\section{Agradecimientos}

Los dibujos fueron preparados por Ester Solís Ugalde. Agradezco profundamente la ayuda brindada por las personas que contribuyeron con este trabajo. Este agradecimiento en particular para todos los investigadores y autores que han contribuido con el conocimiento de los murciélagos de Honduras, particularmente los mencionados en la introducción. Estoy seguro que estarán satisfechos de saber que han contribuido en la elaboración de esta clave. Agradezco a Tim McCarthy (QDDG), Don Wilson, Luis Ruedas, Paúl Velazco, Fiona Reid, Mario Espinal, Lucía I. López y Susana Mora Di Mare por su apoyo en varios aspectos de este trabajo. A Eileen Westwig y Eleanor Hoeger del American Museum of Natural History por las facilidades brindadas para trabajar con la colección de murciélagos. Gracias a Jonathan Hernández por sus sugerencias. La Oficina
Coordinadora de Proyectos de MiAmbiente y el Proyecto Paisajes Productivos proveyeron recursos económicos y logísticos para la preparación de esta clave. Muchas gracias a Daisy Samayoa, Vilma Batista y en especial a Nelson Ulloa y al Señor Ministro José A. Galdámez por su apoyo. Un agradecimiento especial al Icomvis (Instituto Internacional en Conservación y Manejo de Vida Silvestre) de la Universidad Nacional (UNA) de Costa Rica, en particular a Eduardo Carrillo, por su apoyo para culminar este trabajo.

\section{Literatura Citada}

Baird A.B, M.R. Marchán-Rivadeneira, S.G. Pérez y R.J. Baker. 2012. Morphological analysis and description of two new species of Rhogeessa (Chiroptera: Vespertilionidae) from the Neotropics. Occasional Papers, Museum of Texas Tech University 307:1-25. 
Baird, A.B., J.K. Braun, M. A. Mares, J.C. Morales, J. Patton, C.Q. Tran y J.W. Bickham. 2015. Molecular systematic revision of tree bats (Lasiurini): doubling the native mammals of the Hawaiian Islands. Journal of Mammalogy 96(6):1255-1274.

Baker, R.J., S. Solari, A. Cirranello y N.B. Simmons. 2016. Higher level classification of phyllostomid bats with a summary of DNA synapomorphies. Acta Chiropterologica 18:1-38.

Divoll, T.J. y D.G. Buck. 2013. Noteworthy field observations of cave roosting bats in Honduras. Mastozoología Neotropical 20(1):149-151.

Espinal, M. y J.M. Mora y C.M. O'Reilly. 2016. The occurrence of the Peale's free-tailed bat (Nyctinomops aurispinosus, Molossidae) in Central America. Caribbean Journal of Science 49(1):79-82.

Espinal, M. y J.M. Mora. 2016 [2012]. Noteworthy record of Eptesicus brasiliensis (Vespertilionidae) in Honduras. Ceiba 53(2):77-80.

García, J.E. 2009. Breve historia de la Escuela de Biología de la Universidad de Costa Rica (1957-2009). Revista de Biología Tropical 57(Suplemento 1):1-14.

Hernández, D.J. 2015. Programa para la Conservación de los Murciélagos de Honduras (PCMH). p. 41-55. In Rodríguez-Herrera, B. y R. Sánchez (eds.). Estrategia centroamericana para la conservación de los murciélagos. Universidad de Costa Rica, San José, Costa Rica.

Mantilla-Meluk, H. 2014. Defining species and species boundaries in Uroderma (Chiroptera: Phyllostomidae) with a description of a new species. Ocassional papers. Texas Tech University 325:1-29.

Marineros, L. y F. Martínez. 1998. Mamíferos de Honduras. INADES. Tegucigalpa. $374 \mathrm{p}$.

McCarthy, T.J., W.B. Davis, J.E. Hill, J.K. Jones Jr. y G.A. Cruz. 1993. Bat (Mammalia:Chiroptera) Records, Early Collectors, And Faunal List for Northern Central America. Annals of Carnegie Museum 62(3):191-228.

Medellín, R., H.T. Arita y O. Sánchez. 1997. Identificación de murciélagos de México. Clave de campo. Publicaciones Especiales 2. Asociación Mexicana de Mastozoología, A.C., México, D.F. 83 p.

Medina-Fitoria, A., O. Saldaña, J.G. Martínez, Y. Aguirre, W. Silva, M., Chávez, M. Salazar, N. Carballo, O. Jarquín, R.A. González, L. Díaz, C. Chamber, F. Reid, R. Mais, K. Williams, J.M Zolotoff, C. Molina, T. Pérez, J. Rodríguez, L.E. Gutiérrez, M. Fernández. R. Mendieta y J. Pérez. 2015. Nuevos reportes sobre los murciélagos (Mammalia: Chiroptera) de Nicaragua, América Central, con la adición de siete nuevos registros de especies. Mastozoología Neotropical 22(1):43-54.
Mora, J.M. 2012. Lasiurus egregius (Vespertilionidae) in Honduras, Central America. The Southwestern Naturalist 57(1):104-105.

Mora, J.M. y L.I. López. 2012 [2010]. First Record of the Hoary Bat (Lasiurus cinereus, Vespertilionidae) for Honduras. Ceiba 51(2):89-90.

Mora, J.M., L. Marineros y L.I. López. 2014. First record of the striped yellow-eared bat, Vampyriscus nymphaea, (Stenodermatinae, Phyllostomidae) in Honduras. Caribbean Journal of Science 48(1):49-51.

Mora, J.M., M.R. Espinal y L.A. Ruedas. 2016. The Big Free-tailed bat, Nyctinomops macrotis (Gray, 1839), in Central America. Mastozoología Neotropical. En prensa.

OTS (Organization for Tropical Studies). 1971. The book, data, keys, tables, figures and miscellanea. San José, Costa Rica. Mimeografiado.

Reid, F.A. 1997. A field guide to the mammals of Central America \& southeast Mexico. Oxford University Press, New York. 334 p.

Reid, F.A. 2009. A field guide to the mammals of Central America \& southeast Mexico. Second edition. Oxford University Press, New York. 346 p.

Rodríguez-Herrera, B, R. Sánchez y W. Pineda. 2011. First record of Natalus lanatus (Chiroptera: Natalidae) in Costa Rica, and current distribution of Natalus in the country. Ecotropica 17:113-117.

Rodríguez-Herrera, B. y R. Sánchez (eds.). 2015. Estrategia centroamericana para la conservación de los murciélagos. Universidad de Costa Rica, San José. 85 p.

Timm, R.M. y R.K. LaVal. 1998. A field key to the bats of Costa Rica. Occasional Publication Series. University of Kansas Center for Latin American Studies. 22:1-30.

Timm, R.M., R.K. LaVal y B. Rodríguez-Herrera. 2000 [1999]. Clave de campo para los murciélagos de Costa Rica. Brenesia 52:1-32.

Velazco, P.M., y B.D. Patterson. 2013. Diversification of yellow-shouldered bats, genus Sturnira (Chiroptera: Phyllostomidae), in the New World tropics. Molecular Phylogenetics and Evolution 68:683-698

Ziegler, A.C., F.G. Howarth y N.B. Simmons. 2016. A second endemic land mammal for the Hawaiian Islands: a new genus and species of fossil bat (Chiroptera: Vespertilionidae). American Museum Novitates No 3854. American Museum of Natural History, New York. 52 p.

Recibido para publicación el 15 de julio del 2016. Aceptado para publicación el 15 de noviembre del 2016. 